\title{
Bifurcation in Eddy Life Cycles: Implications for Storm Track Variability
}

\author{
ISIDORO ORLANSKI \\ Geophysical Fluid Dynamics Laboratory, Forrestal Campus, Princeton University, Princeton, New Jersey
}

(Manuscript received 14 August 2002, in final form 1 November 2002)

\begin{abstract}
By analyzing a number of very high resolution, nonhydrostatic experiments of baroclinic lifecycles, it was concluded that the intensity of the near-surface baroclinic development influences the upper-level wave to such an extent that it could produce cyclonic or anticyclonic wave breaking. Since the final jet position is equatorward or poleward, the position depends on whether the waves break cyclonically or anticyclonically, respectively. The low-level baroclinicity plays a very important role in the outcome of the wave and feedback to the mean circulation. Using a shallow water model the hypothesis that the intensity of the eddy forcing from the lower layers of the atmosphere can have a profound effect on the disturbances of the upper layers is tested. From these experiments the following is concluded.

For weak intensities, the strong effective beta asymmetries due to the earth's sphericity produce anticyclonic wave breaking and a poleward shift of the zonal jet will occur. For moderate forcing, anticyclonic wave breaking occurs and consequently, as before, a poleward shift of the zonal jet will occur. However, there is an important distinction between weak and moderate forcing. In the latter case, the eddy anticyclonic centers are very intense. The influence of the two anticyclones produces a difluence field that will strain the cyclonic vortex along the SW-NE direction. Consequently, the meridional vorticity flux $v^{\prime} \zeta^{\prime}$ is positive in the north and negative in the south. This process has two effects: thinning the cyclone and producing positive vorticity fluxes on the north, negative fluxes on the south and moving the jet poleward. By increasing the forcing, the cyclone centers become considerably more intense than the anticyclones (CVC) and they are able to deform and thin the anticyclones, thus moving the jet equatorward. This transition is very abrupt; above a threshold amplitude, the life cycle bifurcates to a cyclonic wave breaking.

The implications for storm track variability are quite direct. In normal years, at the entrance of the storm track, intense baroclinicity produces CVCs with a slight shift of the jet equatorward. At the last half of the storm track, due to much weaker baroclinicity, anticyclonic wave breaking occurs (AVCs) displacing the jet poleward. The eddies at the entrance of the storm track develop from the baroclinicity of the subtropical jet. Downstream fluxing and weaker surface baroclinicity make the upper-level waves more aloft and barotropic by the middle of the storm track. These waves normally break anticyclonically, enhancing the subpolar eddy-driven jet. In the warm phase of ENSO, more baroclinicity (and subtropical moisture flux) is present in the eastern Pacific Ocean. This enhanced baroclinicity could support more CVCs in the eastern basin, maintaining the subtropical jet further east.
\end{abstract}

\section{Introduction}

The life cycle (LC) of baroclinic eddies depends greatly on the characteristics of the large-scale flow in which they evolve and, in large part, are responsible for modifying the characteristics of the mean circulation. In the Northern Hemisphere the Pacific and Atlantic storm tracks originate in the vicinity of the stationary troughs over the western part of the respective oceans and terminate near a stationary ridge over the west coast of North America and over Europe. The storm tracks lie downstream and slightly poleward of the vertical and horizontal shear zones associated with the quasi-per-

Corresponding author address: Dr. Isidoro Orlanski, Geophysical Fluid Dynamics Lab, Princeton University, Forrestal Campus, Princeton, NJ 08542.

E-mail: io@gfdl.noaa.gov manent jet streams over Japan and over the east coast of North America. Considerable differences in environmental conditions are observed along the storm tracks. In particular, focussing on the Pacific storm track reveals that the position, intensity, and shape of the local zonal wind, temperature, and moisture have large deviations along the track, from the entrance to the exit. For the most part, these modifications are the product of the mixing action produced by the baroclinic eddies themselves. Large differences are observed due to the interannual variability of the storm tracks; the different behaviors of the baroclinic eddy life cycles are partly responsible for those changes (Lau 1985; Held et al. 1989; Orlanski 1998). Figure 1 shows a schematic of the winter westerly jet axis over the Pacific Ocean for climatology and the two phases of the El Niño-Southern Oscillation (ENSO) cycle.

Weather patterns follow approximately the same tra- 


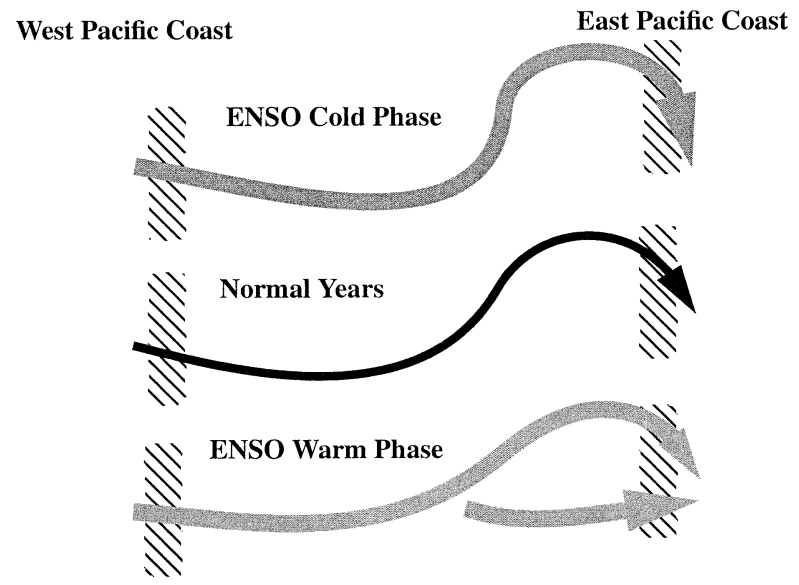

FIG. 1. Schematic diagram of the winter jet over the Pacific Ocean for the ENSO phases and normal years.

jectories as the jet axis along the storm track. In order to describe the characteristics of the interannual variability, let us first examine the normal years. The large zonal variability is associated with a quasi-stationary trough in the first half of the storm track over the western Pacific and a ridge over the second half in the eastern part of the basin. The subtropical jet over Japan at the entrance of the storm track is deflected poleward over the eastern Pacific. In the ENSO warm phase, the entrance trough is slightly weaker as is the ridge at the termination of the storm track. Moreover, the jet seems to split into a subtropical and poleward jet over the eastern Pacific. As a result, the storm tracks are more elongated, thus producing more systems in the warm phase that enter the United States from the southwest rather than from the northwest, which is the case in normal years. In the ENSO cold phase, the eastern ridge is enhanced; consequently, the storm track is further deflected poleward (Orlanski 1998). The weakening of the trough and ridge in the warm events is consistent with the well-known Pacific-North American (PNA) height response of the ENSO cycle (Horel and Wallace 1981). Evidence of the connections between the Tropics and extratropics occurring on longer timescales has been known for many years. For the last 2 decades the influence of tropical heating on the extratropical circulation has been widely investigated by a number of observational and modeling studies (review article, Trenberth et al. 1998). Many of these studies show that heat anomalies like those seen in the equatorial SST during the ENSO cycle can be, through convection and subsidence, a source of Rossby waves. These anomalies can produce a train of Rossby waves that tend to propagate through an equatorial westerly duct in the upper troposphere and produce a response pattern similar to that of the Pacific and North America pattern (PNA) (Hoskins and Karoly 1981; Webster 1981). Trenberth et al. (1998) points out that the climatological stationary planetary waves and associated jet stream, especially in the Northern Hemisphere, can make the total Rossby wave sources somewhat insensitive to the position of the tropical heating that induced them. They also point out that a number of factors influence the dispersion and propagation of Rossby waves through the atmosphere, including zonal asymmetries in the climatological state, transients, and baroclinic and nonlinear effects that can amplify the teleconection response. However, the investigation of the role of the extratropical circulation on the tropical atmosphere and the interannual variability of the interaction has been limited and is not fully understood. A number of issues remain to be answered concerning the midlatitude response to tropical forcing that the quasi-linear explanation of the PNA response to equatorial heating has not been able to explain (Trenberth et al. 1998). We will limit our discussion to a feature that has not been well documented, and that is the effect of the midlatitude high frequencies in the winter storm track being able to amplify the signal of the teleconection. For instance, the splitting jet observed in warm events is not a stationary feature, but rather has scales of weeks, shifting from being a subtropical to a subpolar jet in the eastern Pacific. A stationary heating over the Tropics could not mimic such a transient response. However, it would be feasible to think that the effect of baroclinic eddies can indeed produce such behavior. It is well accepted that the mean meridional circulation in the extratropics, the Farrel cell, is eddy driven. The zonal variability however, could be controlled as well by the action of transient eddies; this issue has not been well understood and we will try to clarify it in this paper.

There is mounting evidence, based on studies of idealized simulations and analysis of the observations, that baroclinic eddies play a very important role in shaping the quasi-stationary circulation (Held et al. 1989; Hoskins and Valdes 1990; Nakamura et al. 1997; Orlanski 1998). In particular, Orlanski (1998) found that baroclinic eddies exert a cyclonic forcing at the western poleward side of the Pacific storm track and anticyclonic forcing at the eastern equatorward side of the storm track. This forcing is consistent with the trough-ridge system that characterizes the winter conditions at the entrance and exit of the storm track.

The atmosphere exhibits a vast range of cyclonic behavior on various time- and space scales. The most extreme cases can be summarized in how they grow and decay. The classical picture of cyclone development by surface baroclinicity of the environment has been extended to include very common observed cases of growth by fluxing energy from a system upstream. This process is known as "downstream baroclinic development" (Simmons and Hoskins 1979; Orlanski and Katzfey 1991). Downstream baroclinic development can produce wave packets that are occasionally observed in the atmospheric circulation (Lee and Held 1993; Chang 1993; Berbery and Vera 1996, among others). The life cycle of baroclinic eddies could terminate by fluxing 
energy to a system downstream or by more violent wave breaking by nonlinear critical layer absorption or by other means. Although some eddy life cycles require the interaction of many modes, particularly those characterized by downstream development (Orlanski and Gross 2000), most of our understanding of the eddy life cycle has emerged from studies examining normal modes in zonally symmetric jets. Nonlinear life cycle experiments using the faster growing linear baroclinic normal modes on a realistic basic zonal flow exhibit many of the characteristics of observed midlatitude storms (e.g., Simmons and Hoskins 1978, 1980; Balasubramanian and Garner 1997a,b, among others). Observational studies have concluded that the basic structure of midlatitude baroclinic eddies are indeed very similar to those of nonlinear baroclinic normal modes (Lim and Wallace 1991; Chang 1993).

An intriguing possibility concerning the mechanism for baroclinic normal modes to decay was presented by Simmons and Hoskins (1980) and Thorncroft et al. (1993, hereafter THM). They found two canonical stages of wave breaking, LC1 and LC2. LC1 is the classical picture they describe of nonlinear critical layer absorption in which the wave breaks anticyclonically. In the later stages of the wave, the upper-level trough is being thinned by the effects of the anticyclone that advects the temperature southwestward. To quote the authors, "The whole process is an example of what might be called equatorward Rossby wave breaking by analogy with the more common cases of planetary-scale Rossbywave breaking in the wintertime middle stratosphere" (e.g., Clough et al. 1985; McIntyre and Palmer 1984). LC2, on the other hand, grows in a manner similar to $\mathrm{LC} 1$; what is remarkable is the completely different way the latter evolves. In the LC2 case, the cyclonic wrapup on the polar side of the jet, which also occurred early in $\mathrm{LC} 1$, continues to expand to a much larger scale and never breaks anticyclonically. LC2 decays very slowly whereas LC1 zonalizes very fast. Consequently, LC2 is left with a robust cyclone and an undulated potential vorticity (PV) pattern for a very long time.

Although THM does not present a clear discussion on the eddies affecting the mean circulation, they do discuss the possibility that a storm track could have either type of cyclone development. In both cases the cyclones start on the polar side of the jet. As they evolve, LC1 shifts south of the jet continually thinning due to the anticyclonic shear. On the other hand, LC2 remains poleward of the jet axis. Actually, in THM, Fig. 3 shows the initial and end profiles of the zonal jet for both cases. We can very clearly see a large shift in latitudinal position of the jet in both cases. LC1 ends up with a jet poleward of the initial position, and LC2 has a jet slightly southward of the initial position. THM used two different initial jets in order to obtain these two different evolutions (LC1 and LC2). A barotropic cyclonic shear was added in LC2 to modify the position of the upperlevel critical layers and the intensity of the index of refraction. That was the basic contention of their conclusion needed to explain the different behaviors of LC1 and LC2.

These results raise some interesting questions. For example, can the frequent occurrence of either of these two canonical states (cyclonic and anticyclonic wave breaking) be the cause for the variability of the storm track (as shown in Fig. 1)? Is it possible to have more types of LC1 in the normal or cold phases of ENSO that shift the jet axis poleward or more LC2 types in the warm phase that could explain the shift to the south? Orlanski (1998) analyzed the Pacific storm track for a 10 -yr period, 1980 to 1990, that includes two ENSO cycles. It was found that although the positions of the entrance jets were similar in these two periods, the baroclinicity extended more eastward in El Niño years. Complementary to these results is a case study by Shapiro et al. (2001) that found that in the period of 199799, the warm phase of ENSO supports LC2 development whereas in the cold phase LC1 was more prevalent. There is a possibility then, that the environmental conditions could support either of the life cycles (LC1 and/ or LC2). In order to answer these questions several baroclinic life cycle experiments were performed. In the next section we will briefly discuss the three dimensional simulations of baroclinic life cycles for a variety of basic states. In section 3, we present the forced shallow water global model used for a number of upperlevel life cycle simulations. The results of the global wavenumber 7 response for different forcing parameters will be presented in section 4 . Global wavenumbers 5 , 6,7 , and 8 are examined in section 5. The summary and discussion will be presented in section 6 .

\section{Three-dimensional simulations of baroclinic life cycles}

Solutions were obtained by integrating the nonhydrostatic, fully compressible model (ZETANC ${ }^{1}$; an early version of the model is described in Gross 1997) with very high resolution $(5$ and $10 \mathrm{~km}$ in the middle of the channel) and different initial states that portray different storm track environments. Fourteen experiments have been designed to use profiles for a diversity of jet configurations, wide and narrow jets with strong and weak vertical shears, in dry and moist atmospheric conditions. Analyses of these baroclinic life cycle simulations will be published elsewhere but let us summarize some of the results relevant to this discussion.

Figure 2 shows the zonal mean jet and eddy kinetic energy (EKE) after 13 days of the evolution of the baroclinic eddies, for both dry and moist conditions. Figure $2 \mathrm{a}$, corresponds to the dry case and Fig. $2 \mathrm{~b}$ is for the

\footnotetext{
${ }^{1}$ The ZETANC model, developed at the Geophysical Fluid Dynamics Laboratory (GFDL) by Steve Garner, is a high-resolution nonhydrostatic fully compressible model of hemispheric extent (see http://www.gfdl.noaa.gov/ io/Bubble.html).
} 

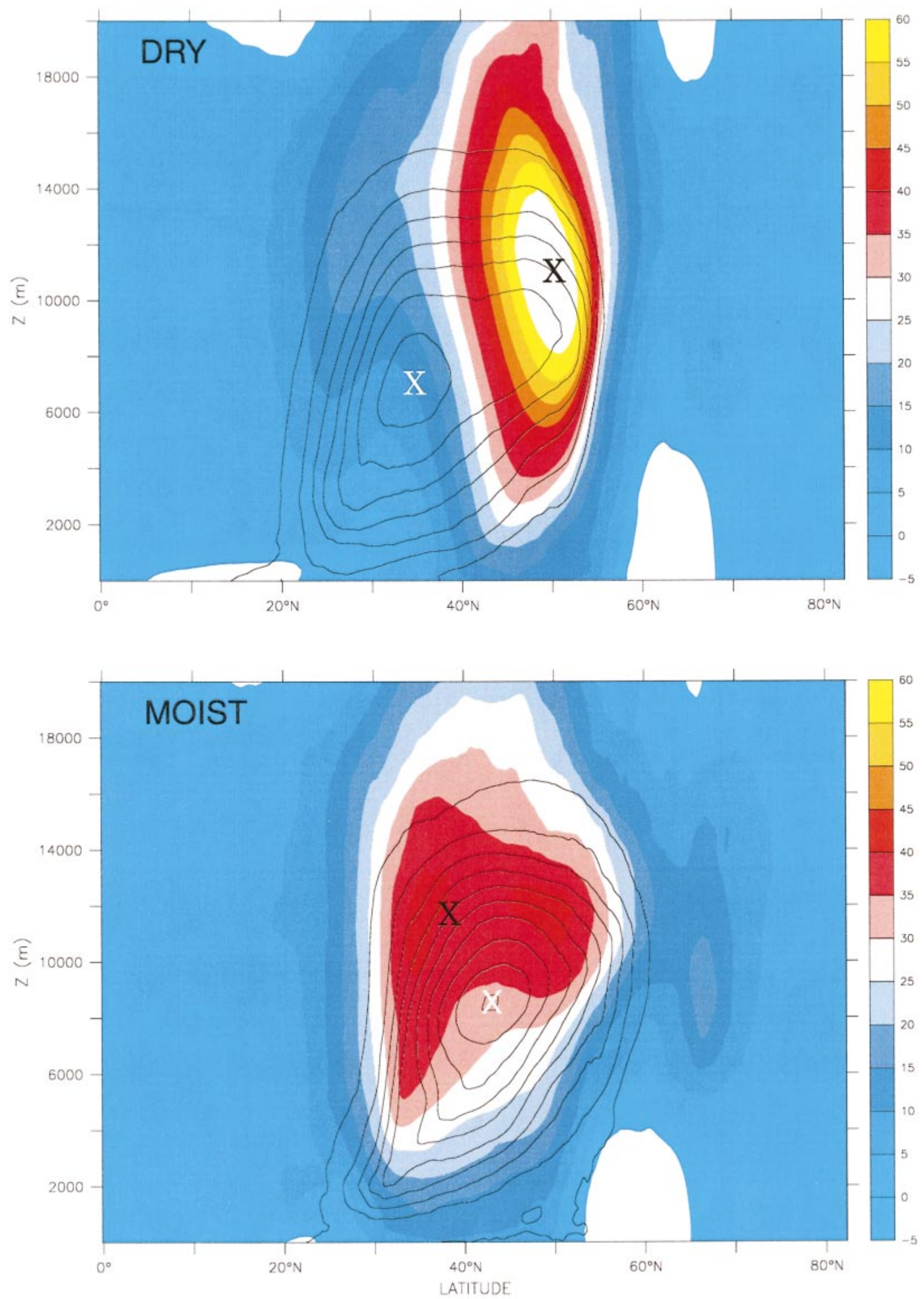

FIG. 2. Cross section of zonal mean flow (color bands) and EKE (black contours) at day 13 for the three-dimensional high-resolution nonhydrostatic simulations. (a) Dry atmosphere and (b) moist atmosphere. The solutions run for 15 days and both dry and moist cases are started with the same initial conditions of basic state and perturbations. However, in the moist case there is a moisture distribution only in the tropical latitudes. 
moist environment. Since the initial moisture distribution was concentrated in the low latitudes, both dry and moist solutions have the same development for the first few days. The differences in the zonal jet for both cases after 13 days is quite remarkable. The dry solution has the final jet displaced to the north $\left(50^{\circ} \mathrm{N}\right)$ of the initial position $\left(45^{\circ} \mathrm{N}\right)$, whereas the moist solution has it to the south $\left(38^{\circ} \mathrm{N}\right)$. The maximum eddy kinetic energy is located south of the jet maximum in the dry case and to the north in the moist case. This is very similar to that found by THM (their Fig. 3) for the LC1 and LC2 evolution. A clearer description of the time evolution of the zonal jet for these two solutions can be seen in Fig. 3. The solutions correspond to a initial jet with a $20^{\circ}$ latitude width and a maximum wind speed of $70 \mathrm{~m}$ $\mathrm{s}^{-1}$ (narrow jet with strong vertical shear). Figure $3 \mathrm{a}$ shows the evolution of the zonal mean jet $(8000 \mathrm{~m})$ and the eddy kinetic energy over its life cycle for the dry atmosphere and Fig. 3b for a moist atmosphere with otherwise identical conditions. The solutions correspond to a single wavenumber $(m=7)$. As expected the moist solution is more energetic than the dry one. The first 4 days are practically identical. Since the moisture distribution is larger in low latitudes and practically decays to very low values by $50^{\circ} \mathrm{N}$, active convection takes place only after the frontal system associated with the wave has advected enough moisture to the middle latitudes to make a substantial departure from the evolution of the dry wave. Clearly, by the end of the life cycle, the dry jet is located poleward of its initial position whereas the moist solution jet is located a few degrees southward of its initial position.

These results have strong similarities with the LC1 for the evolution of the dry case and the LC2 evolution with the moist case. However, the results seem to contradict THM conclusions that both life cycles were different because the characteristics of the upper-level index of refraction and critical layer in their study were distinctly different. In this case, the dry and moist solutions have started with the same upper-level distributions. It also seems to disprove the suggestion by Balasubramanian and Garner (1997a) that the difference in life cycles was connected to the initial normal mode structure. Both dry and moist solutions are initially very similar in structure and the zonal flow characteristics are identical. In these two cases, the baroclinicity in the later part of the life cycle is different due to the advection of moisture by the wave. The moist case being more intense, could break cyclonically, whereas the weaker dry case breaks anticyclonically. These results are confirmed by most of the other solutions that we analyzed. Cases with the same baroclinicity, even in a dry atmosphere can change from an LC1 evolution to an LC2 evolution by only removing the surface drag. Without surface drag, for strong shears, the dry solutions could be very cyclogentically intense and also could displace the jet southward similar to the moist case. On the other hand, weak shears even without surface drag similar to that used by THM, develop as an LC1. Our contention, then, is that it is the intensity of the lowerlevel wave that will determine the outcome of the upperlevel wave; if it is very intense, the upper level will break cyclonically whereas for a weaker system, it will break anticyclonically.

We have reduced the problem to a very simple question: "Could the intensity of the lower-level eddies determine the outcome of the upper-level flow?' To unequivocally answer this question, we used a shallow water model to simulate the evolution of large-amplitude upper-level waves. If the model is forced to simulate the stretching produced by the lower-level waves, it should be able to show under which conditions the waves so generated could break cyclonically or anticyclonically. For a relevant simulation of the evolution of the upper-level wave with a shallow water model, we should first inspect the distribution of the height of an isentropic surface (300 K; see Fig. A1) for the 3D simulations discussed previously. This isentrope was selected because it is characteristic of the separation between the lower and the upper atmosphere (around $z \sim$ $5000 \mathrm{~m}$ ). This solution corresponds to the moist baroclinic solution (see Figs. $2 \mathrm{~b}$ and $3 \mathrm{~b}$ ). Also, the potential vorticity for that surface and the vertically averaged relative vorticity anomaly (with respect to the zonal mean) between two levels $(5-10 \mathrm{~km})$ are shown in Fig. A1. Note the wave-like shape of the anomaly height $(300 \mathrm{~K})$ has an amplitude close to $2000 \mathrm{~m}$, whereas the potential vorticity is showing signs of the upper wave rolling up. Also, it is worth noting the large amplitude of the vertically averaged relative vorticity anomaly with a maximum close to $1.75 \times 10^{-4} \mathrm{~s}^{-1}$ and a minimum of $-0.75 \times 10^{-4} \mathrm{~s}^{-1}$; these differences between cyclonic and anticyclonic vorticities will be more relevant when discussing the evolution of the shallow water model solutions.

\section{A Global Shallow Water Model ${ }^{2}$}

\section{a. The 11/2-Layer Shallow Water Model $\left(S M_{1 / 2}\right)$}

The Global Shallow Water Model is a versatile spectral model with a tracer option ideal for simulating the global circulation where a low-order model is justified. Since the model is spectral (triangular truncation), the prognostic variables are relative vorticity, divergence, and height. The momentum equations from which the vorticity and divergence equations were derived are shown Eqs. (A.4)-(A.8). The model conserves the potential vorticity, $\mathrm{PV}$, and is

$$
\mathrm{PV}=\frac{\zeta(\lambda, \phi, t)+2 \Omega \sin (\phi)}{g^{\prime} h(\lambda, \phi, t)-g^{\prime} h_{f}(\lambda, \phi, t)},
$$

\footnotetext{
2 The Flexible Model System (FMS) offers the Global Shallow Water Model developed by Isaac Held (see http://www.gfdl.noaa. gov).
} 

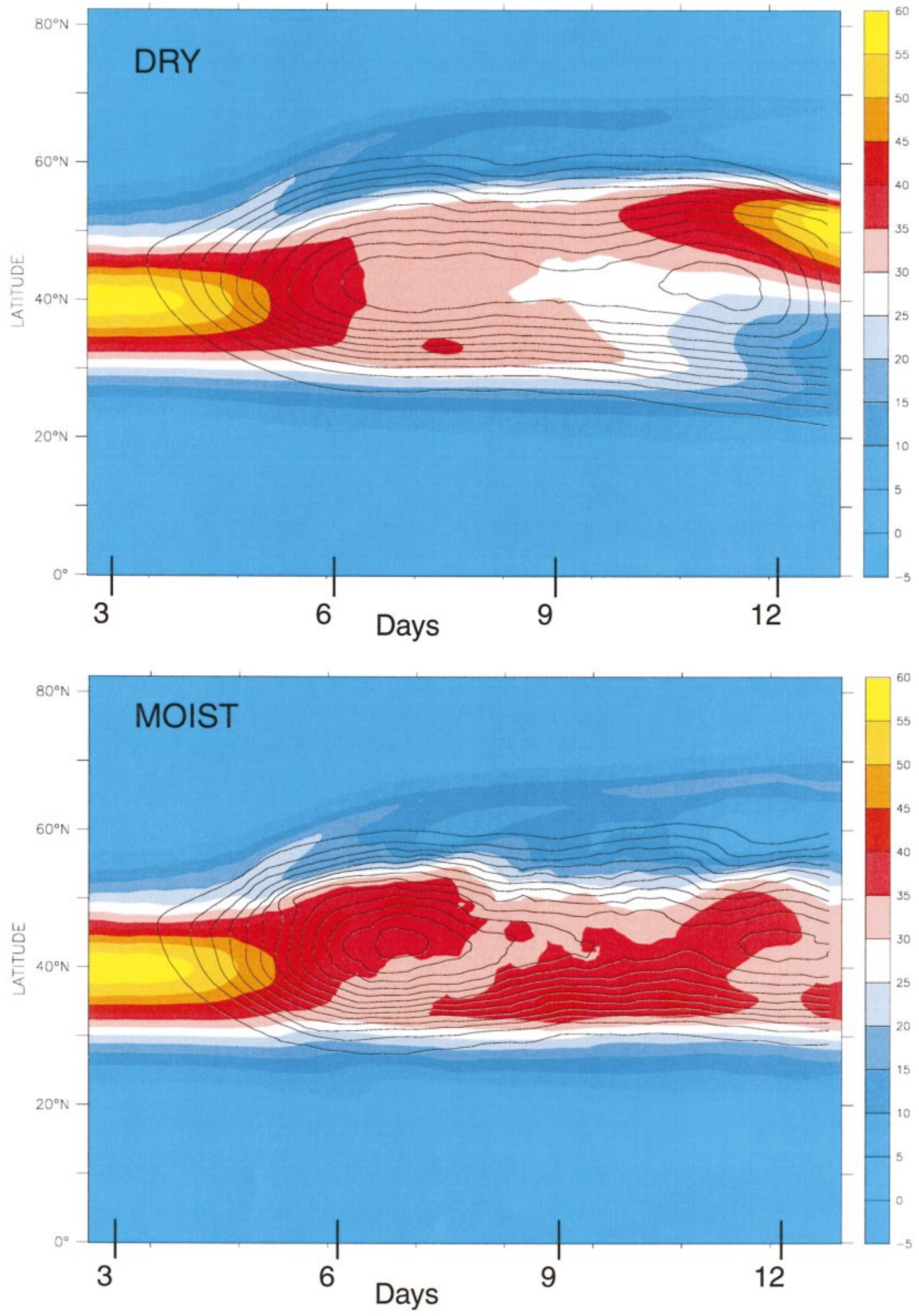

FIG. 3. Evolution of the zonal mean flow (color bands) and eddy kinetic energy (black contours) for the solutions shown in Fig. 2 at $z=8000 \mathrm{~m}$. The contour interval of eddy kinetic energy is $200 \mathrm{~m}^{2} \mathrm{~s}^{-2}$. 
where $\zeta$ is the relative vorticity, $2 \Omega \sin \phi$ is the Coriolis parameter, $g^{\prime}$ is the reduced gravity, $h$ is the height of the dynamic layer, and $h_{f}$ is the height of the lower layer where the forcing is applied. We are using two versions of the shallow water model and presenting corresponding solutions whose characteristics we describe in the appendix. The first one is the shallow water model (hereafter, $\mathrm{SM}_{1 \frac{1}{2}}$ shown in Fig. A2b). The forcing $h_{f}$ [h2 in Eq. (A.10)] is a prescribed function of space and time that tends to mimic the stretching effects due to the unstable lower-level wave [(Eq. (A.11)] as shown in Fig. A1:

$$
\begin{aligned}
& h_{f}=\bar{h}_{f}(\phi)+A(t) h_{w}, \\
& h_{w}=W \frac{U(\phi)-c}{U_{0}-c} \cos \left[m_{0}(\lambda-c t)\right], \quad \text { if } U(\phi)-c \geq 0,
\end{aligned}
$$

and

$h_{w}=0$

$$
\text { if } U(\phi)-c<0 \text {. }
$$

The first rhs term of (3.2) represents a mean slope of the lower interface (see appendix). The second term is the prescribed wave forcing that mimics the baroclinic evolution from the lower layer. As (3.2) indicates, $h_{w}$ is given as a disturbance that propagates in the zonal direction with phase speed $c$ and wavenumber $m_{0}$. The latitudinal variation is the same as the zonal jet profile; $h_{w}$ is different than zero only in the region where the jet is larger than $c$ (simulates the confinement by the critical layers). The amplitude that modulates the wave forcing $A(t)$ and, in simple terms, represents the time evolution of the lower-level wave is

$$
A(t)=\left(\frac{t}{t_{\mathrm{LF}}}\right)^{2} \exp \left[-\left(\frac{t}{t_{\mathrm{LF}}}\right)^{2}\right]
$$

The maximum value of the forcing of $t=t_{\mathrm{LF}}$ (the timescale of the forcing life cycle) is: $A\left(t=t_{\mathrm{LF}}\right)=e^{-1}$.

Then, prescribing the expression $h_{f}$ from Eq. (3.2) in the thickness equation,

$$
\frac{d h}{d t}+h \boldsymbol{\nabla} \cdot \mathbf{V}=-\frac{\partial h_{f}}{\partial t}+\boldsymbol{\nabla} \cdot h_{f} \mathbf{V}+\nu \Delta^{n} h .
$$

The first two rhs terms provide the forcing for the $\mathrm{SM}_{1 \frac{1}{2}}$ model and the last term is the hyperdiffusivity, which, for this case, we use either $n=4$ or 8 with $\nu \sim 1 \mathrm{~h}$. Conservation of potential vorticity is a nice property of $\mathrm{SM}_{1 \frac{1}{2}}$. Although we are adding mass sources and sinks from (3.2), there is not a net mass added to the system; if it were, PV should still be conserved. There is however a serious drawback of this forcing to simulate the evolution of an unstable upper-level wave: for weak forcing the solutions are quite realistic and well behaved; however, since our interest is to explore the evolution of the waves in a highly nonlinear regime, $h_{f}$ in $\mathrm{SM}_{1 \frac{1}{2}}$ is like imposing a corrugated bottom topography. Therefore, in order to have a large wave response, the imposed forcing should have large amplitudes. Consequently, Rossby-wave radiation and nongeostrophic dynamics make the response uncontrollable (see appendix for more discussion). A simpler variation of this forced model can render more controllable solutions when generating large finite-amplitude waves.

\section{b. Shallow Water Model (SM)}

The model SM as shown in Fig. A1c is very similar to $\mathrm{SM}_{1 \frac{1}{2}}$ with an important difference. Equations (A.4)(A.8) are the same in both models; the only difference is in the treatment of the forcing in Eq. (3.4). Instead of the $\mathrm{SM}_{1 \frac{1}{2}}$ forcing in Eq. (3.4), SM has a Newtonian restoration of the perturbation height $h^{\prime}$ to the height of the wave forcing $h_{w}$ [Eq. (3.2)]. Similar mass sources have been introduced in studies on stratospheric dynamics (Polvani et al. 1995; Sobel and Plumb 1999, among others).

The SM height equation with the Newtonian restoration is

$$
\frac{d h}{d t}+h \boldsymbol{\nabla} \cdot \mathbf{V}=-\frac{1}{\tau_{R}} A(t)\left(h^{\prime}-h_{w}\right)+\nu \Delta^{n} h .
$$

The first term on the rhs is the Newtonian restoration of $h^{\prime}$, the deviation of the instantaneous zonal mean $h$. We used $6 \mathrm{~h}$ for the restoration time constant $\tau_{R}$. Restoring the perturbation height to $h_{w}$ has the desirable effect of producing a wave that is very similar to the upper-level waves even in strong baroclinic developments. Since the initial development of baroclinic instability requires that the upper and lower wave disturbances should be phase locked, forcing SM with this kind of restoration term seems to mimic the phase-locking process very well. The solutions of SM do not seem to produce strong Rossby-wave radiation in the initial period of development as in $\mathrm{SM}_{1 \frac{1}{2}}$ (see discussion and Fig. A3 in the appendix). However, this model also has a major limitation. The restoration term in Eq. (3.5) can be a source or a sink of PV. We estimated the amount of PV in the solutions, and those values tend to be small-only a few percent of either the meridional advection of PV or the divergence of meridional PV flux [see Sobel and Plumb (1999) for a thorough discussion on the relation of mass sources like this one and PV in shallow water models]. We are confident about the robustness of these solutions. Similar regimes that will be discussed in the following sections were also found with other models, that is, the full 3D simulations, the $\mathrm{SM}_{1 \frac{1}{2} \text {, }}$, and simple barotropic models. For this reason, we chose to present the bulk of the results obtained with SM; however, similar solutions from $\mathrm{SM}_{1 \frac{1}{2}}$ displayed the change in zonal mean winds (see Fig. A4).

The initial jet used for this study is similar to that used by Simmons and Hoskins (1980), THM and our wide jet in the three-dimensional simulations (section 2 ), and is given by 
Maximum Value of Area Averaged Eddy Kinetic Energy

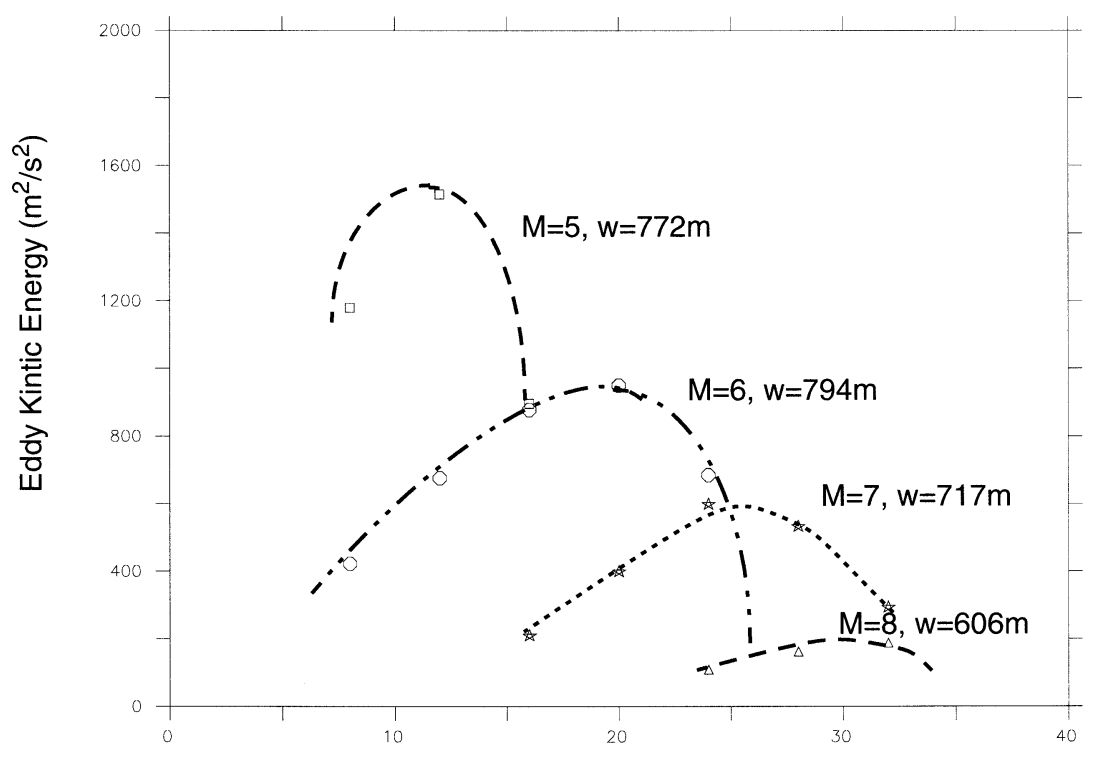

FIG. 4. Eddy kinetic energy response for four different wavenumbers $(m=5,6$, 7, and 8 ) as a function of the forcing phase speed for the SM solutions.

$$
U(\phi)=U_{0} \sin \left[\pi \sin (\phi)^{2}\right]^{3} .
$$

We tested different parameter ranges for the phase velocities, values of $\boldsymbol{t}_{\mathrm{LF}}$ and values of $U_{0}$. We found that the behavior of the solutions is qualitatively the same; all are very sensitive to the forcing amplitude $\boldsymbol{W}$, the wavenumber $\boldsymbol{m}$, and $\boldsymbol{c}$, but less sensitive to the values of the other parameters. The values of the forcing coefficients are

$$
\begin{aligned}
U_{0} & =40 \mathrm{~m} \mathrm{~s}^{-1}, \quad t_{\mathrm{LF}}=4 \text { days, and } \\
m & =8,7,6, \text { and } 5 .
\end{aligned}
$$

We will use $\boldsymbol{W}$ (in meters) as the forcing amplitude for the rest of the paper. Figure 4 shows the maximum value of the area-averaged eddy kinetic energy (EKE) as a function of different values of $\boldsymbol{c}$ and wavenumber $\boldsymbol{m}$, for different constant $\boldsymbol{W}$ s. For a given $\boldsymbol{m}$, the variation of EKE with $\boldsymbol{c}$ is similar for different values of $\boldsymbol{W}$; the larger the $\boldsymbol{W}$, the higher the values of EKE will be. However, the maximum value of EKE is obtained for the same $\boldsymbol{c}$ regardless of the value of the forcing amplitude $\boldsymbol{W}$. The value of $c$ that maximizes the EKE depends on the initial jet profile and in particular the meridional extension of the forcing that is proportional to $\boldsymbol{U}-\boldsymbol{c}$. Although one is tempted to consider only those cases with the value of $c$ that maximizes the response, that would not necessarily be justified. This is because the baroclinic wave evolution that these simulations try to portray may not propagate with this optimal value of $\boldsymbol{c}$.

The relative vorticity response as a function of the forcing amplitude is shown in Fig. 5 for $m=7$ and a constant $c=24 \mathrm{~m} \mathrm{~s}^{-1}$ (the optimal $c$ for $m=7$ ). In this figure, two pair of curves are shown for each of the five forcing amplitudes, the cyclonic vorticity maximum and the anticyclonic vorticity minimum. Notice that for a weak forcing $(269 \mathrm{~m})$ the positive and negative vorticity have a symmetric evolution. However, for a stronger forcing, the system becomes more asymmetric; the cyclonic vorticity becomes more intense reaching values a few times larger than the Coriolis frequency (six times the Coriolis parameter in the stronger forcing). The anticyclonic vorticity on the other hand, levels off at approximately $-f$, or the absolute vorticity at zero. Later we will see that these strong asymmetries between the cyclonic and anticyclonic evolution are of paramount importance in the different wave evolutions. Let us start our discussion for the response of wavenumber $m=7$, the same wavenumber used in the threedimensional simulation shown in Figs. 2 and 3.

\section{Wavenumber 7}

\section{a. Potential vorticity}

The potential vorticity for $3,6,8$, and 16 days in the life cycle of the wave is shown in Fig. 6. The forcing $(269 \mathrm{~m})$ is weak and the evolution is very similar to a classical baroclinic normal mode decay. The blue lines, for reference, are the linear critical layers due to the basic flow. The wave trough tends to grow and tilt in the NE-SW direction; as it becomes thinned by the anticylonic shear of the basic flow, it tends to move west around the anticyclone. Finally, with the hyper diffusion and reabsorbing in the high PV area north of the jet, the flow zonalized with a slight displacement of $2^{\circ}$ to the north. The entire solution seems weakly non- 


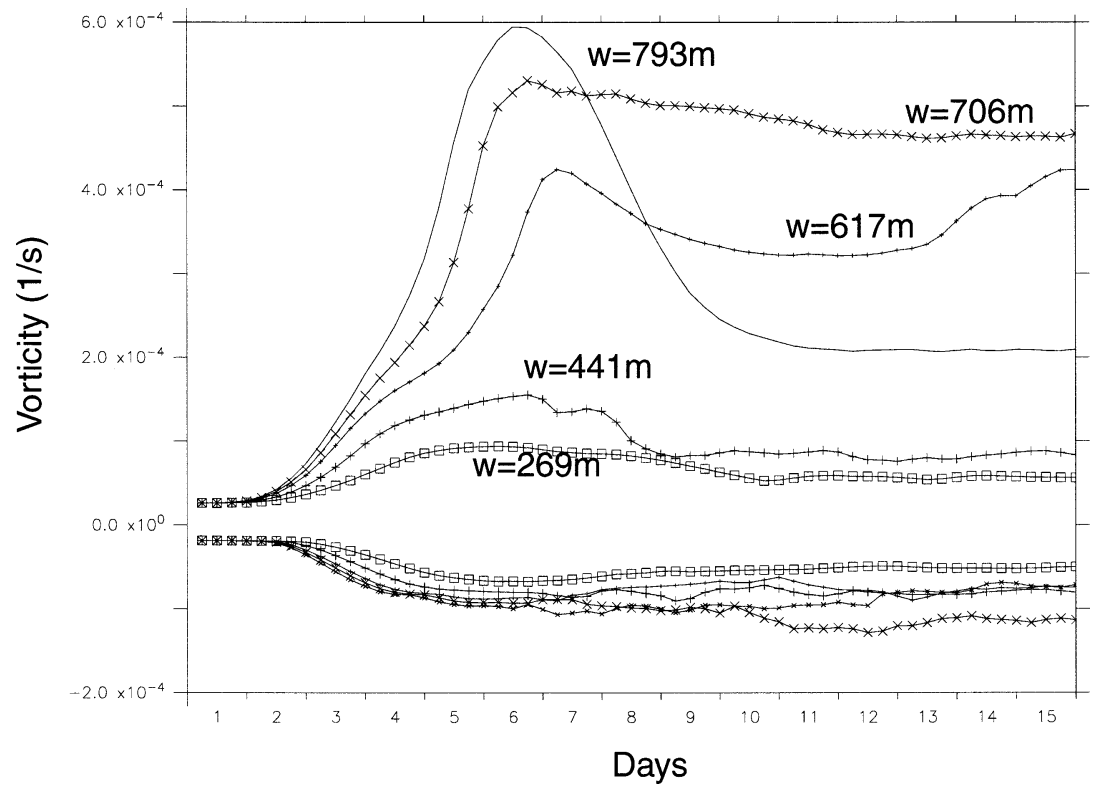

FIG. 5. Evolution of the maximum positive and minimum negative vorticity response for $m$ $=7$ and $c=24 \mathrm{~m} \mathrm{~s}^{-1}$ at different forcing amplitudes.

linear and the wave breaking could have been classified as anticylonic, similar to that described for stratospheric Rossby waves that propagate equatorward and are absorbed by a critical layer (Clough et al. 1985; McIntyre and Palmer 1984; THM).

Figure 7 shows the PV response to different forcing amplitudes at a time when the response had attained approximately the maximum amplitude (Fig. 5). Figure 7 shows a remarkable shift from anticyclonic wave breaking to cyclonic wave breaking. The final response after 16 days is not only remarkable but dynamically rich. By contrast, the two weak forcing cases zonalized to a flow that has been displaced poleward of the original jet. The two stronger cases produce a jet south of the initial position and maintain the propagation of strong cyclonic vortices (like modons). It seems clear that the mechanism acting on the LC1 and LC2 life cycles is also operating in these solutions, insofar as these are realistic portraits of the evolution of upper-level baroclinic wave development.

\section{b. Asymmetries between cyclonic and anticyclonic development}

There are a few mechanisms that can break the symmetry of cyclonic and anticyclonic development (Fig. 5). For instance, the stretching term being proportional to the absolute vorticity will produce more intense cyclones than anticyclones. This is perhaps the major difference between quasigeostrophic dynamics that produce symmetric centers with the primitive equations or even semigeostrophic dynamics that include the full stretching term (Hoskins 1971). The asymmetry between cyclonic and anticyclonic vorticity generations is due to the fact that, given the same amount of convergence and divergence, in principle due to mass conservation, the absolute vorticity and consequently the stretching term will be larger for positive relative vorticity (we will argue later for slight asymmetries in the divergence centers due to the sphericity of the earth). Figure 5 shows the maximum and minimum vorticity generated in the five experiments. It is clear that, at the beginning, small amplitudes, both positive and negative, grow similarly; this is particularly true for small forcing (e.g., $W=269 \mathrm{~m}$ ). However, for stronger forcing, as both centers (positive and negative) grow, the asymmetry becomes important. At the point when the absolute vorticity and PV is zero, the stretching term will be zero $(\zeta=-f)$ and negative vorticity cannot grow further negative due to stretching. This means that, where the cyclonic centers could continue to grow, the anticyclones reach a saturation level where no further growth is possible by this mechanism. Actually, even if other forcing could generate a more negative vorticity, this would make the absolute vorticity less than zero and would allow for an inertial instability that could equilibriate the minimum vorticity to the local $-f$ or the absolute vorticity close to zero.

A word on the positive vorticity; the maximum of strong forcing exceeds 4 or 5 times the local $f$. It seems rather excessive, however these values have been reached in the high-resolution baroclinic simulations discussed in section 2. Note that because the circulation around the cyclone should be the same as that around the anticyclonic center, the case in which the positive vorticity is much larger than the magnitude of the negative vorticity, the cyclone area will be proportionally smaller than the anticyclonic (see Fig. 6). The behavior 

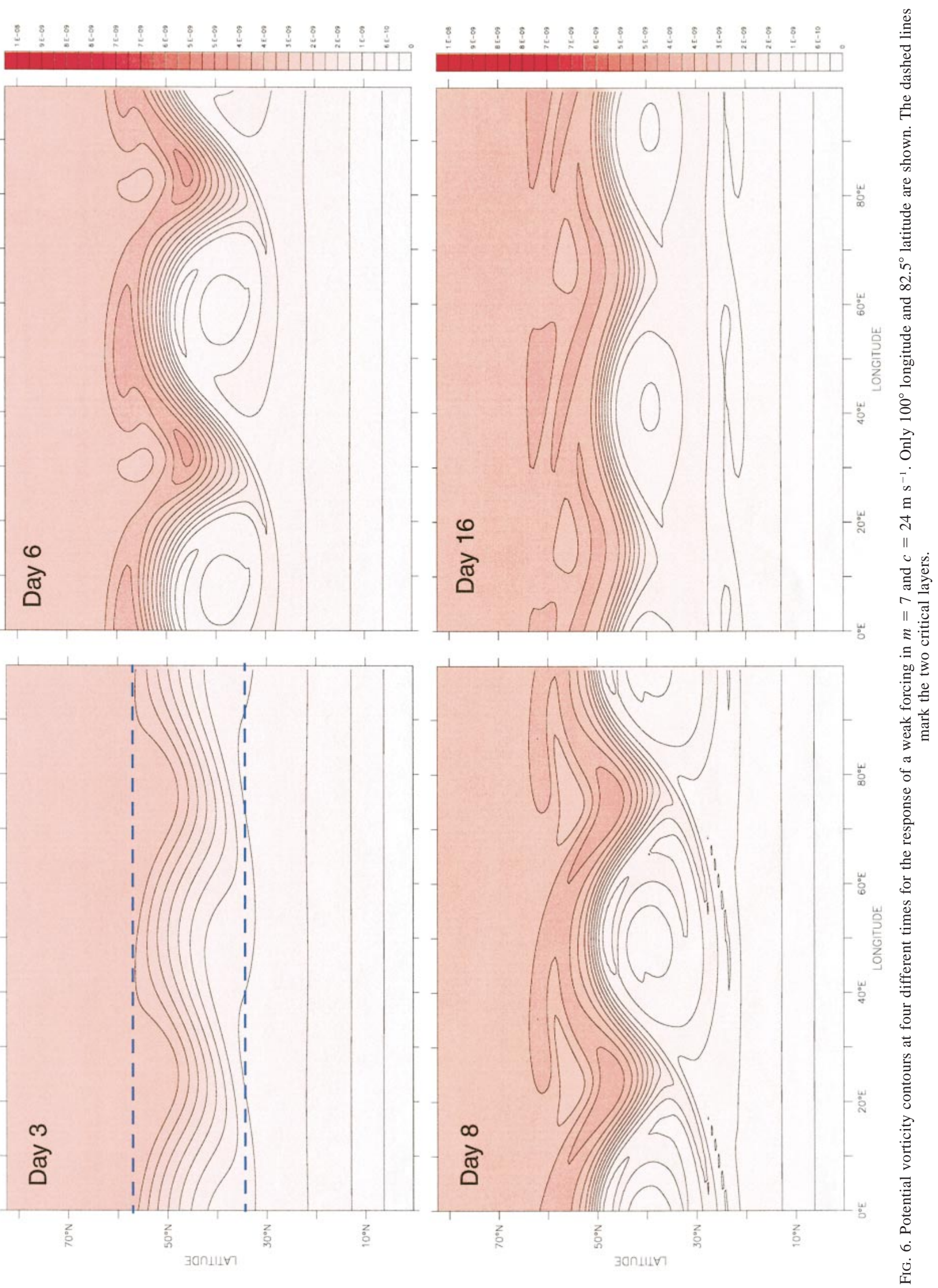

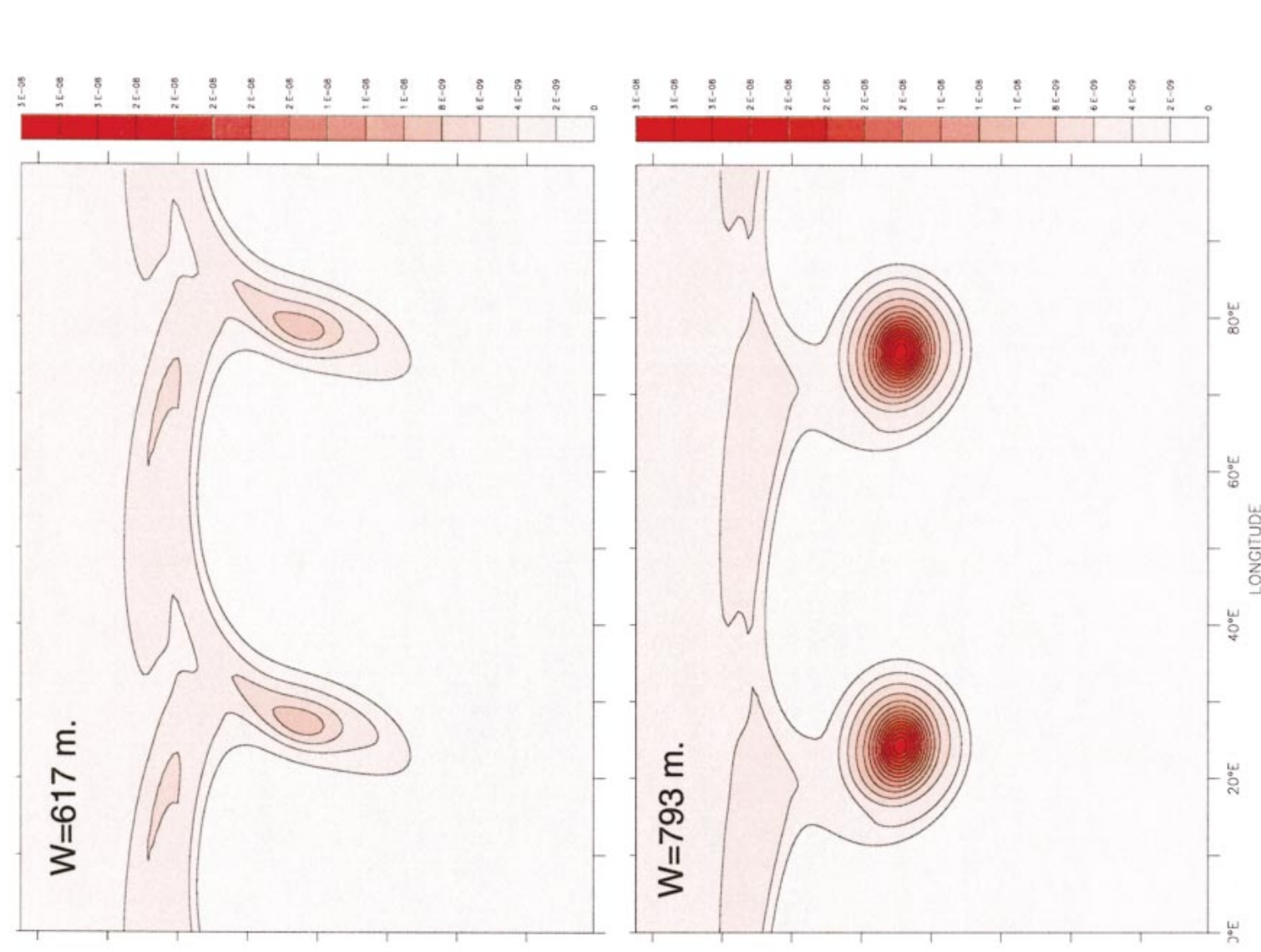

$\frac{1}{6}$
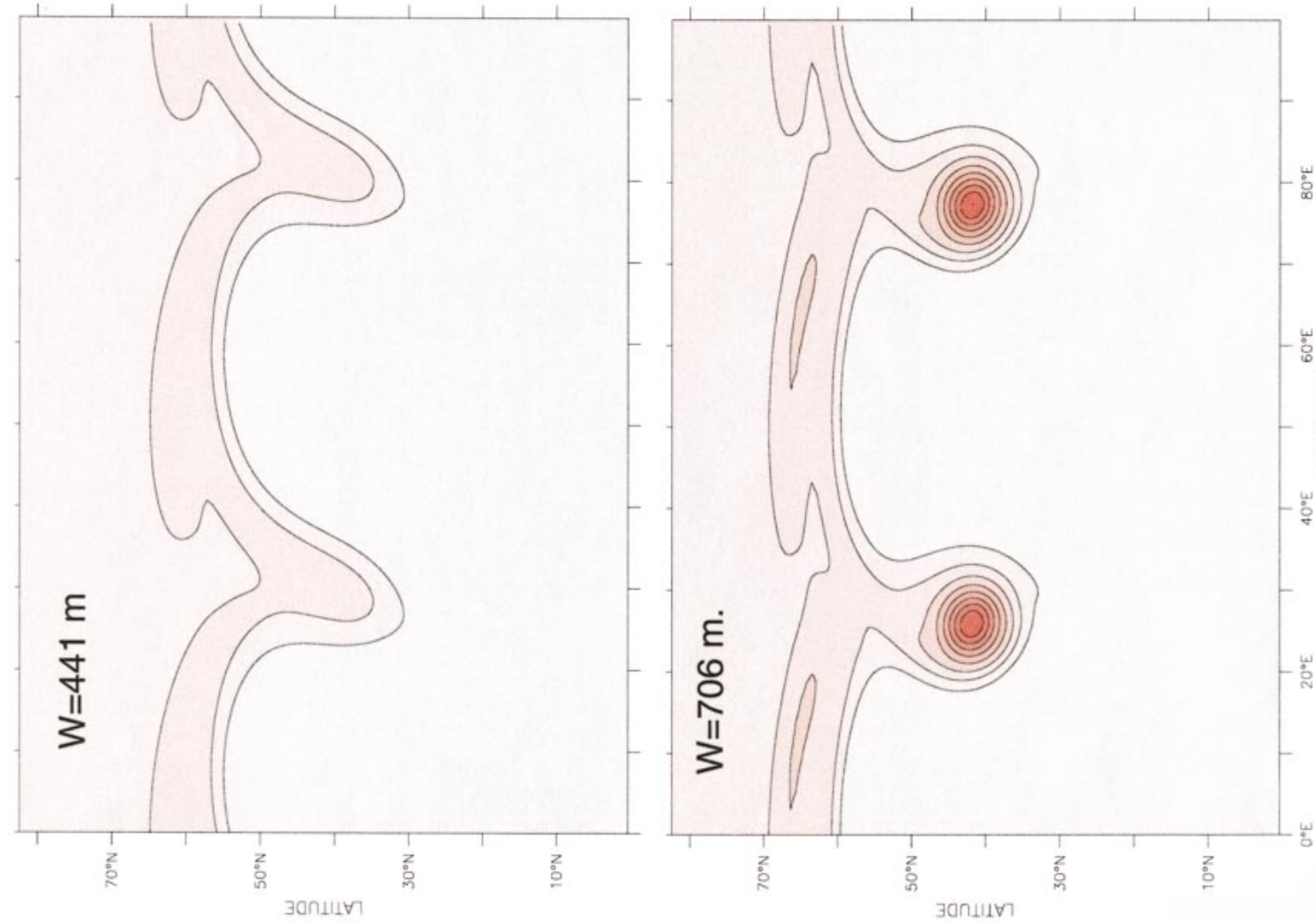

.0

हี

ป ส

0

突要要

可

全

ㄷำ

.

峑总离

可

㟧

言

흔

言

苛 


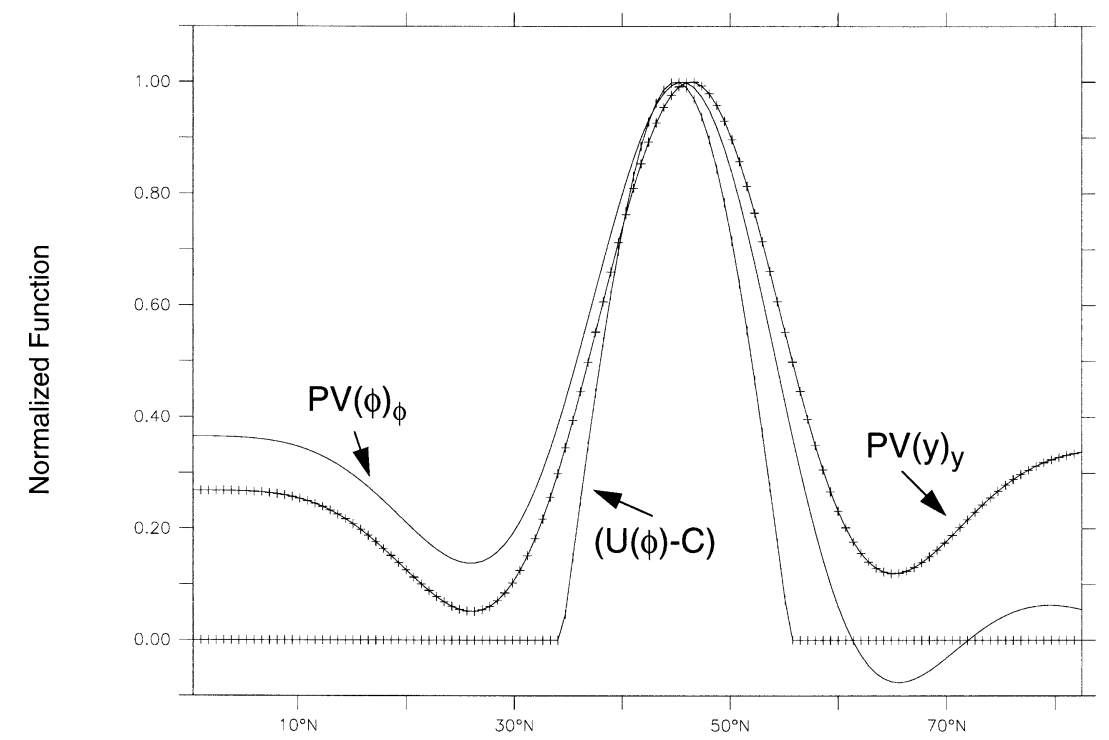

FIG. 8. The meridional PV gradient of the basic state, the profile of $U-c$ and the meridional $\mathrm{PV}$ gradients assuming no metric terms and the Coriolis parameter linear with latitude. In order to show them in the same graph, each was normalized by its maximum value.

of the cyclonic centers for most of the solutions tends to grow and decay in time. However, solutions like $W$ $=706 \mathrm{~m}$ and, to some extent but differently, $W=617$ $\mathrm{m}$ (as we will discuss in a following section) have reached an equilibration with their environment that can sustain these centers quite permanently. Let us first describe why weak forcing seems to be biased toward anticyclonic wave breaking.

\section{c. Anticyclonic wave breaking for weak forcing}

A number of studies attempted to discuss the difference in wave breaking for baroclinic waves in cartesian (cyclonic wave breaking) or spherical (anticyclonic wave breaking) geometries (Whitaker and Snyder 1993; Balasubramanian and Garner 1997a,b). Whitaker and Snyder (1993) use arguments based on a two layer simulation of nonlinear baroclinic development. The meridional displacement of cyclones and anticyclones tends to strengthen the anticyclones and weaken the cyclone in spherical geometry. They suggested that the asymmetry was produced by the metric terms associated with the variation in length rather than the curvature of the latitude circles in the nonlinear stage of the wave. Balasubramanian and Garner (1997a), on the other hand, proposed that the asymmetry was due to the fact that the normal modes in Cartesian and spherical geometries are different and could determine their final different evolutions.

Our results, cannot totally support either assumption. As we have shown in the three-dimensional initial value simulations, the dry and moist solutions have the same evolution for the initial periods (few days) and then as nonlinearity takes place, they depart in their evolution.
Moreover, our forcing in the shallow water model that portrays the effects of low-level baroclinic development has some unrealistic features. The forcing has the maximum and minimum divergence at the same latitude, whereas in a true baroclinic wave, due to the poleward heat fluxes, the maximum and minimum divergence are meridionally displaced as required to validate the Whitaker and Snyder (1993) suggestion. The basic state in this model has very asymmetric effective beta $(\mathrm{PV})_{\phi}$ due to the metric term in the vorticity shown in Fig. 8. It is considerably higher in the southern latitudes $\left(\sim 30^{\circ} \mathrm{N}\right)$ than at the poleward latitudes $\left(\sim 60^{\circ} \mathrm{N}\right)$. The symmetric shape of the jet shown in Fig. $8,[U(\phi)-$ $C]$ will be the same as the shape of the eddy forcing [Eq. (3.3)]. It seems then that in the neighborhood of the critical layers, $U(\phi)-C \sim 0$, the disturbances will feel the influence of the effective beta, that being higher on the southern critical layer will produce more westward tendencies relative to the north. This effect, for weak disturbances, will tilt the eddies NE-SW as seen in the wave evolution in Fig. 6. For completeness of argument, the meridional gradient of $(\mathrm{PV})_{y}$, as if in a Cartesian system (without the metric term in the vorticity and linear variation of $f$ with latitude), is plotted in Fig. 8. The effective beta for the Cartesian geometry shows an opposite asymmetry to that of $(\mathrm{PV})_{\phi}$. Following the previous argument, the disturbances in these cases will tilt NW-SE with a possible cyclonic wave breaking. The difference in the structure of the normal modes already may contain the asymmetry of the effective beta, which favors the Balasubramanian and Garner's (1997a) suggestion for weak developments. However, for strong developments there is a complete redistribution of PV due to the large amplitude attained 


\section{Anticyclonic Vortex Control (AVC)}

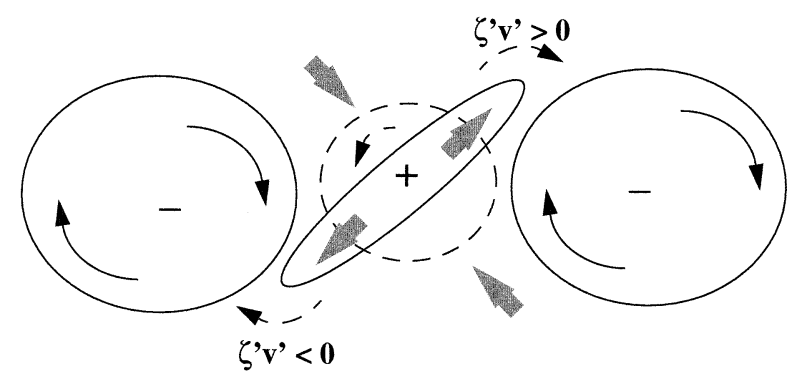

Cyclonic Vortex Control (CVC)

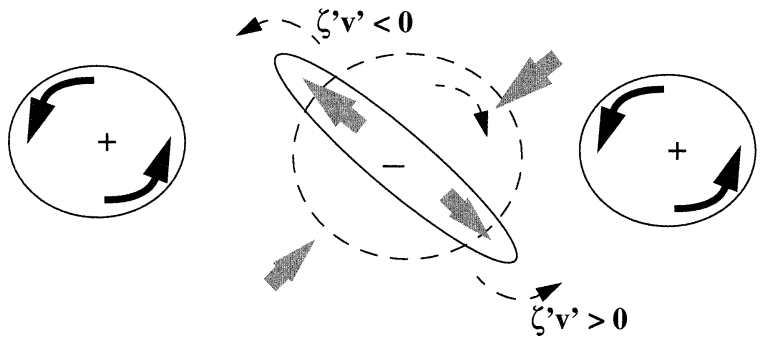

FIG. 9. Schematics of vortices interaction of both signs. (a) A stronger negative vortex center is shown, and (b) a stronger positive vortex center is shown. Solid black arrows indicate the circulation; bold grey arrows indicate the direction of deformation. The meridional vorticity fluxes that this interaction will produce is also indicated.

by the eddies and quasi-linear arguments may not suffice.

\section{d. Strong interactions, and anticyclonic and cyclonic wave breaking}

The simple observation of the vortex intensity displayed in Figs. 7c,d precludes us from making quasilinear arguments about their evolution. In fact, it may be difficult to interpret their different evolutions and breaking on the basis of the lack of refraction index and critical layers (as in THM), even if those arguments could be based on nonlinear dynamics. It is, however, a very tractable scenario to think about the mechanism of vortex interactions. Figure 9 shows a sketch of two intense vortices of one sign interacting with a weaker vortex of the opposite sign. Let us first discuss the case with the stronger anticyclonic centers. The upper graph in Fig. 9 shows two large anticyclones interacting with a weaker cyclone in the center. The influence of the two anticyclones produces a difluence field that will strain the cyclonic vortex along the SW-NE direction. Consequently, the meridional vorticity flux $v^{\prime} \zeta^{\prime}$ is positive in the north and negative in the south. This discussion will be relevant when we discuss the effects on the zonal flow. We called that state anticylonic vortex control (AVC). The reverse situation is shown in the lower graph. Here, the cyclonic vortices are more intense than the anticylonic vortex. The anticyclone under the influence of the stronger cyclones, will elongate in the NWSE direction producing meridional vorticity fluxes negative on the north and positive on the south. This case, of course, is the cyclonic vortex control (CVC). Figure 10 shows the vorticity distribution and instantaneous circulation at $t=6$ days for the solutions shown in Figs. $7 \mathrm{~b}$ and $7 \mathrm{~d}$. The upper figure corresponds to the case in which the AVC mechanism is operating and the lower graph shows the vorticity for when CVC is dominant. Notice the corresponding tilt in NE-SW direction for AVC and the NW-SE direction for CVC. Clearly the geopotential distribution for these two cases will be the classical picture of anticyclonic and cyclonic wave breaking with poleward momentum for the first case and equatorward momentum for the second case. The wind vectors for both situations suggest what final distribution the zonal mean jet will have: a jet center at about $60^{\circ} \mathrm{N}$ for the case with $W=617 \mathrm{~m}$ and around $35^{\circ} \mathrm{N}$ for the case with $W=793 \mathrm{~m}$. It seems that AVC and CVC represent two canonical mechanisms by which strong interactions are accomplished. It will be shown that the two mechanisms AVC and CVC cover a wider range of situations, two of which are LC1 and LC2.

\section{e. Changes in the zonal mean wind}

The long-term consequence of the different life cycles can be measured by the effect left on the zonal mean wind. In simple terms, the zonal average of the longitudinal component of the wind $U$ is quite sensitive to the eddy meridional vorticity fluxes because the evolution of the zonal wind is

$$
\frac{\partial}{\partial t} \bar{U}=\bar{v}(\bar{\zeta}+f)+\overline{v^{\prime} \zeta^{\prime}}+D .
$$

The first term on the right is the meridional flux due to the mean meridional velocity, and the second term is the meridional flux due to the eddies and $D$ represents dissipation. The relative vorticity is given by

$$
\zeta=\frac{1}{a \cos \phi} \frac{\partial}{\partial \lambda} v-\frac{1}{a \cos \phi} \frac{\partial}{\partial \phi}(u \cos \phi) .
$$

Note that the zonal mean eddy meridional vorticity fluxes are the same as the zonal mean divergence of the meridional momentum fluxes. The Fig. 9 schematics show that AVC will have the eddy meridional vorticity fluxes positive on the poleward side of the eddies and negative on the equatorward side, thus producing a westerly acceleration on the poleward side and easterly acceleration to the south of the eddy axis. The reverse will be true for the CVC. Figure 11 confirms these findings where the evolution of $\bar{U}$ and eddy meridional vorticity fluxes as a function of time for two forcing am- 

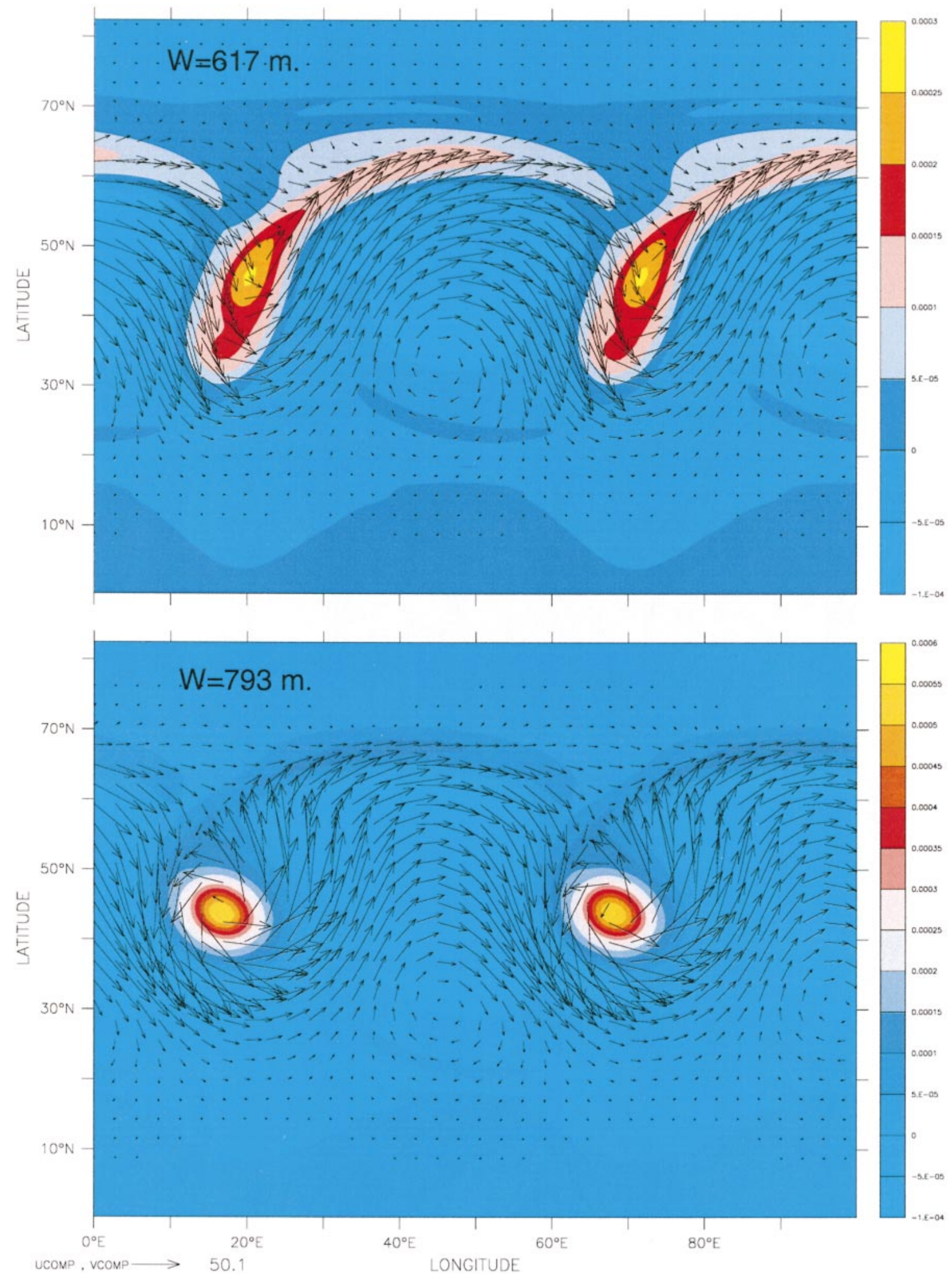

FIG. 10. Relative vorticity and circulation vectors that illustrate the two processes AVC $(W=617 \mathrm{~m})$ and CVC $(W=793 \mathrm{~m})$. Both solutions are for $m=7$ and $c=24 \mathrm{~m} \mathrm{~s}^{-1}$. 

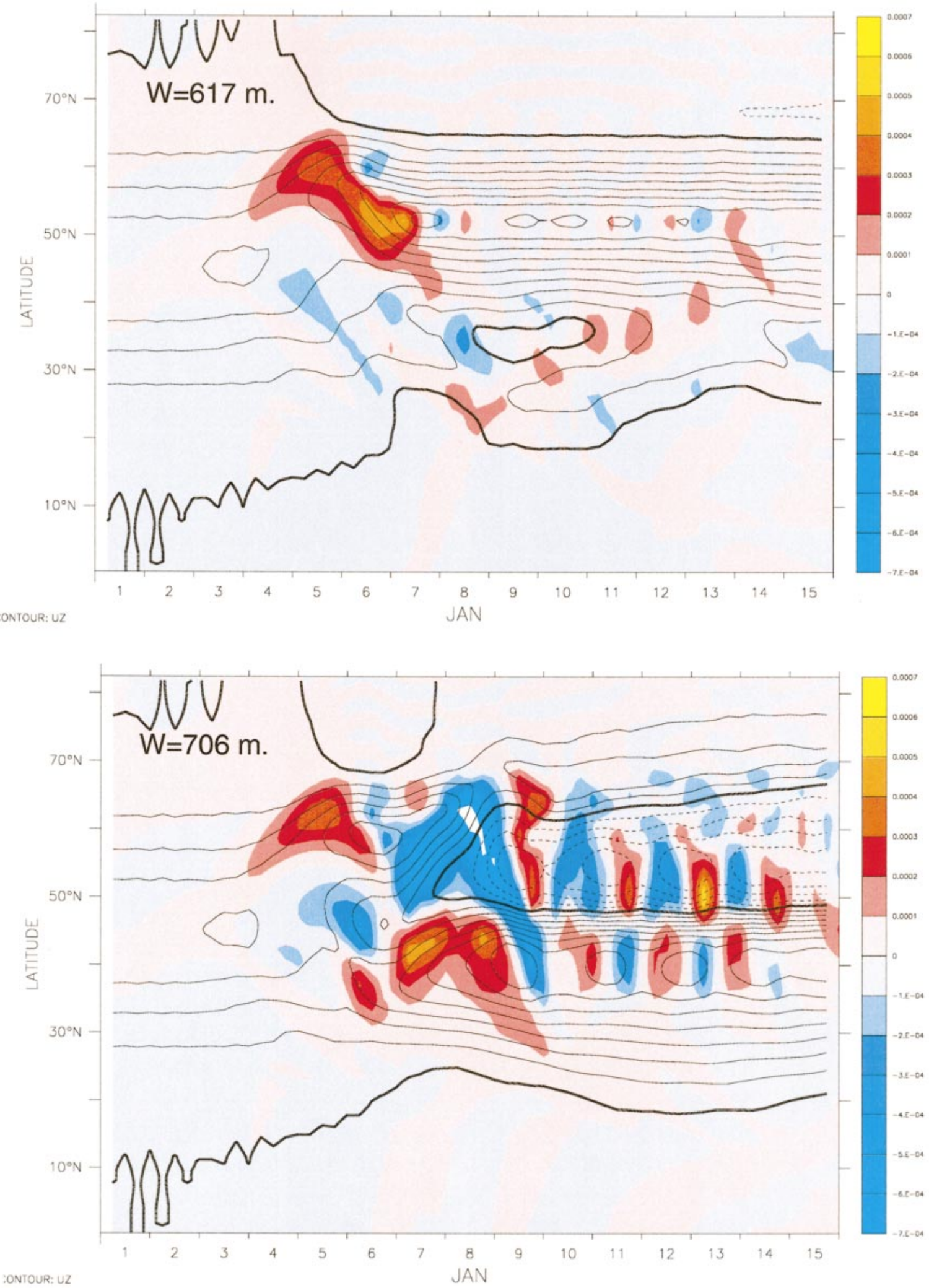

FIG. 11. Evolution of meridional eddy vorticity fluxes (color bands) and resultant change in the zonal flow (black contour, positive for solid lines and dashed for negative values; the contour interval is $10 \mathrm{~m} \mathrm{~s}^{-1}$ ) for both cases of Fig. 10 (see text). 
plitudes $W=617 \mathrm{~m}$ and $706 \mathrm{~m}$ are shown. In this figure, the color shading corresponds to the meridional vorticity fluxes, which, as Eq (4.1) indicates, is an important term for changing the zonal momentum. Figure 11a, for $W$ $=617 \mathrm{~m}$, shows that by day 5 there is a large positive anomaly on the north side of the jet and a slight negative anomaly on the south side. This meridional vorticity flux produces a strong shift in the zonal mean wind to the north of the initial jet axis. This behavior is consistent with the mechanism of AVC acting. A very different development can be seen (Fig. 11b) for the stronger forcing $W=706 \mathrm{~m}$. As in the previous case, the vorticity fluxes begin as positive northward and negative southward, and even temporarily shift the zonal jet poleward because, as previously discussed, for weak amplitudes there is an anticyclonic bias. However, by day 6 , since the eddies keep increasing in amplitude, the system turns over and we notice a strong shift in the fluxes, intensely negative to the north and positive to the south. This produces a change in the zonal mean jet-a displacement to the south of the initial jet axis. Clearly, the forcing intensity that translates to the eddy response intensity is responsible for shifting the jet from poleward to equatorward. The poleward (AVC) and equatorward (CVC) displacement for both cases is clearly shown in this figure. A complementary picture of these two cases is provided in Figs. 12a,b. The evolution of $U$ and eddy kinetic energy as a function of time are shown for the case $W=617 \mathrm{~m}$ (Fig. 12a) and $W=$ $706 \mathrm{~m}$ (Fig. 12b). Eddy energy moves south and decays by day 11 in the AVC case, whereas the CVC case produces quite permanent eddy activity for the entire 15-day period. This eddy activity is basically composed of cyclonic centers that have been equilibrated with a new distribution of zonal PV. [A comparison with the solutions obtained with $\mathrm{SM}_{1 \frac{1}{2}}$ (that conserves layer PV) forced by a bottom topography type is shown in Fig. A4]. The solutions display the same characteristics shown in Fig. 12-an abrupt change in the zonal jet final position. For moderate forcing, the jet is positioned poleward of the initial jet, whereas, for more intense forcing, the jet shifts to the equatorward side of the initial position. Let us return to the solutions of SM.

Figure 13 shows a summary of all the cases (13) with different values of $\boldsymbol{c}$, and $\boldsymbol{W}$ for $m=7$. Figure 13a shows the maximum eddy kinetic energy over the entire life cycle versus the latitudinal position of the final zonal jet maximum for each given case. Figure $13 \mathrm{~b}$ is similar to Fig. 13a but shows the potential enstrophy. Figure 13 is paramount to our conclusions; it shows that as the intensity of the eddies increases, the jet moves further and further to the north by means of AVC. But there is a threshold level of eddy kinetic energy or PV anomaly that for eddies surpassing that level, there is bifurcation on the eddy life cycle changing from AVC to CVC and also on the zonal mean circulation. The flow enters into a different regime due to CVC; the jet shifts to the south of the initial position. Not only do the jet and the zonal
PV change, but eddies could equilibrate to a final amplitude that can propagate undisturbed in the modified PV field.

\section{f. The possibility of generating permanent cyclones centers}

As previously mentioned, under special conditions for large amplitude, the eddies equilibrate into permanent propagating cyclonic eddies. Those eddies have drastically changed the mean zonal flow (see Fig. 12b) and its PV distribution. These possible long-lived systems are not only a feature of the shallow water model solution since THM and our own three-dimensional simulations show similar cyclone behavior. We have run the shallow water model for those solutions for 30 days without an apparent decrease of cyclone strength. To illustrate the characteristics of solutions with permanent cyclones (modones), which differ from the other solutions, let us first discuss the conditions that may lead to such structures. Since PV is conserved, at least after the forcing has disappeared, the solution with permanent propagating cyclones should satisfy

$$
\frac{d}{d t} \mathrm{PV}=\left(u-C_{\mathrm{ph}}\right) \frac{\partial}{\partial x} \mathrm{PV}+v \frac{\partial}{\partial y} \mathrm{PV}=0,
$$

where $C_{\mathrm{ph}}$ is the propagating velocity of the vortex centers and should be distinguished from the propagation velocity that we imposed to the forcing $\boldsymbol{c}$. It will become apparent soon that the two are different and the ability to generate the permanent cyclones is not due to any resonance condition $\left(c=C_{\mathrm{ph}}\right)$. Although the full velocity $(u, v)$ is given by rotational and divergent components, for these cases the rotational component is much larger than the divergent component (not shown) and Eq. (4.3) could be simplified by assuming that $u$ and $v$ are derived from the streamfunction alone. In that case, Eq. (4.3) can be rewritten as

$$
J\left(\psi+C_{\mathrm{ph}} y, \mathrm{PV}\right)=0,
$$

where the Jacobian is expressed by $\Psi$ the total stream function, $y$ (is the latitude times earth's radius $y=a \phi$ ) and total PV. To nontrivially satisfy Eq. (4.4), PV = $F\left(\Psi+C_{\mathrm{ph}} y\right)$. First let us inspect the dependence of PV with the streamfunction $\Psi$ for three solutions shown as $\mathrm{a}, \mathrm{b}$, and $\mathrm{c}$ in Fig. 13a. The solutions $a$ and $b$ (not shown) have a rather incoherent relation between PV and $\Psi$. However, the solution (point c) that displays the permanent cyclones, is remarkably different. Figure 14a shows the scatterplot of potential vorticity versus streamfunction for the corresponding point $\mathrm{c}$ in Fig. 13a. It seems clear from the graph that a very coherent relation between PV and $\Psi$ exists for this case.

The plot is done utilizing all points over the last half life cycle of the eddies (7 days). Although the relation shown in this graph seems quite compact, Eq. (4.4) implies that PV is not only a function of $\Psi$ but $\Psi+C_{\mathrm{ph}} y$, where $C_{\mathrm{ph}}$ should be somehow determined by inspecting 

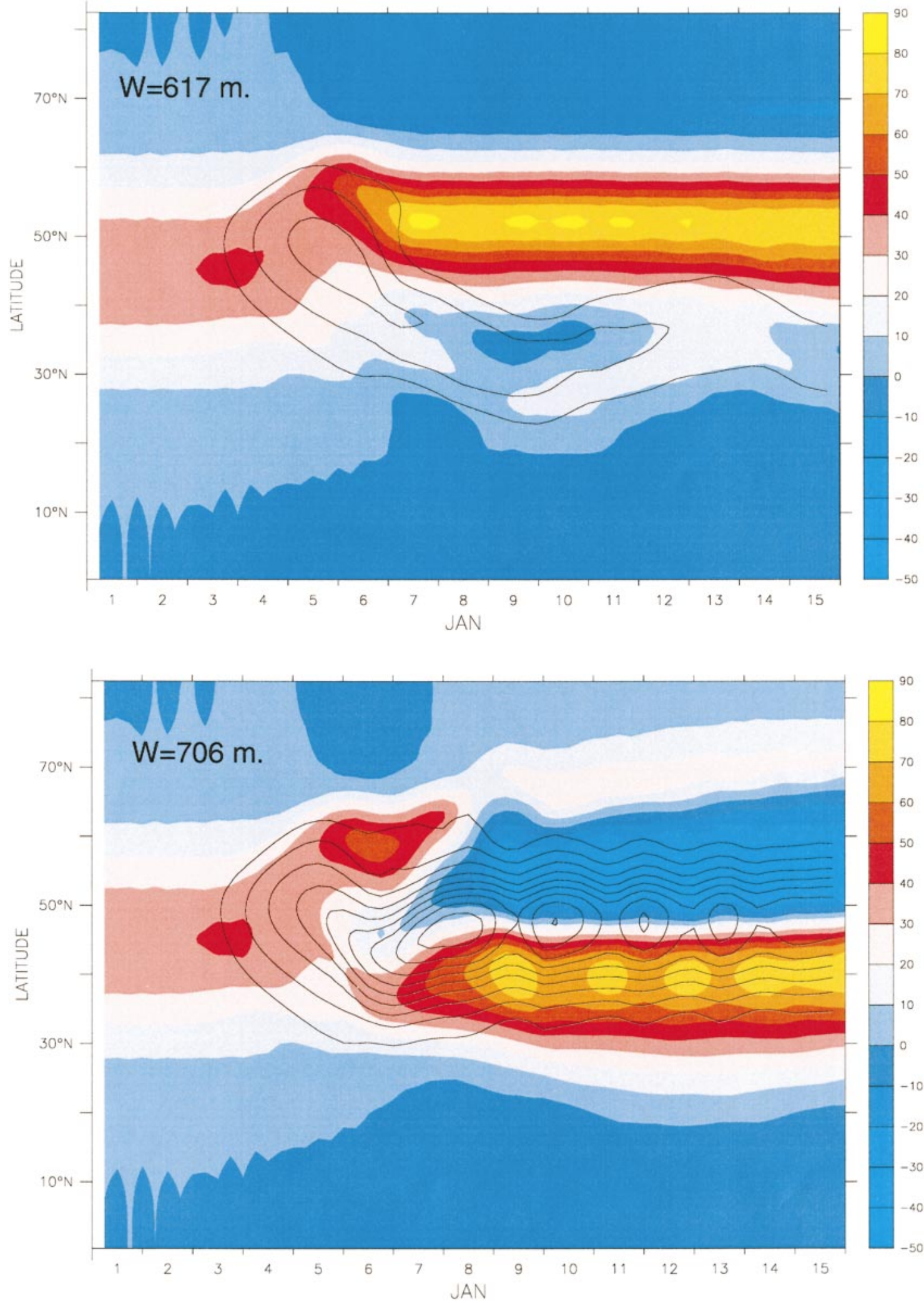

FIG. 12. Similar to Fig. 11. The evolution of the zonal mean flow (color bands) and the EKE (black contour, interval 100 $\mathrm{m}^{2} \mathrm{~s}^{-2}$ ). This graph should be compared with the three-dimensional solutions of Fig. 3. 

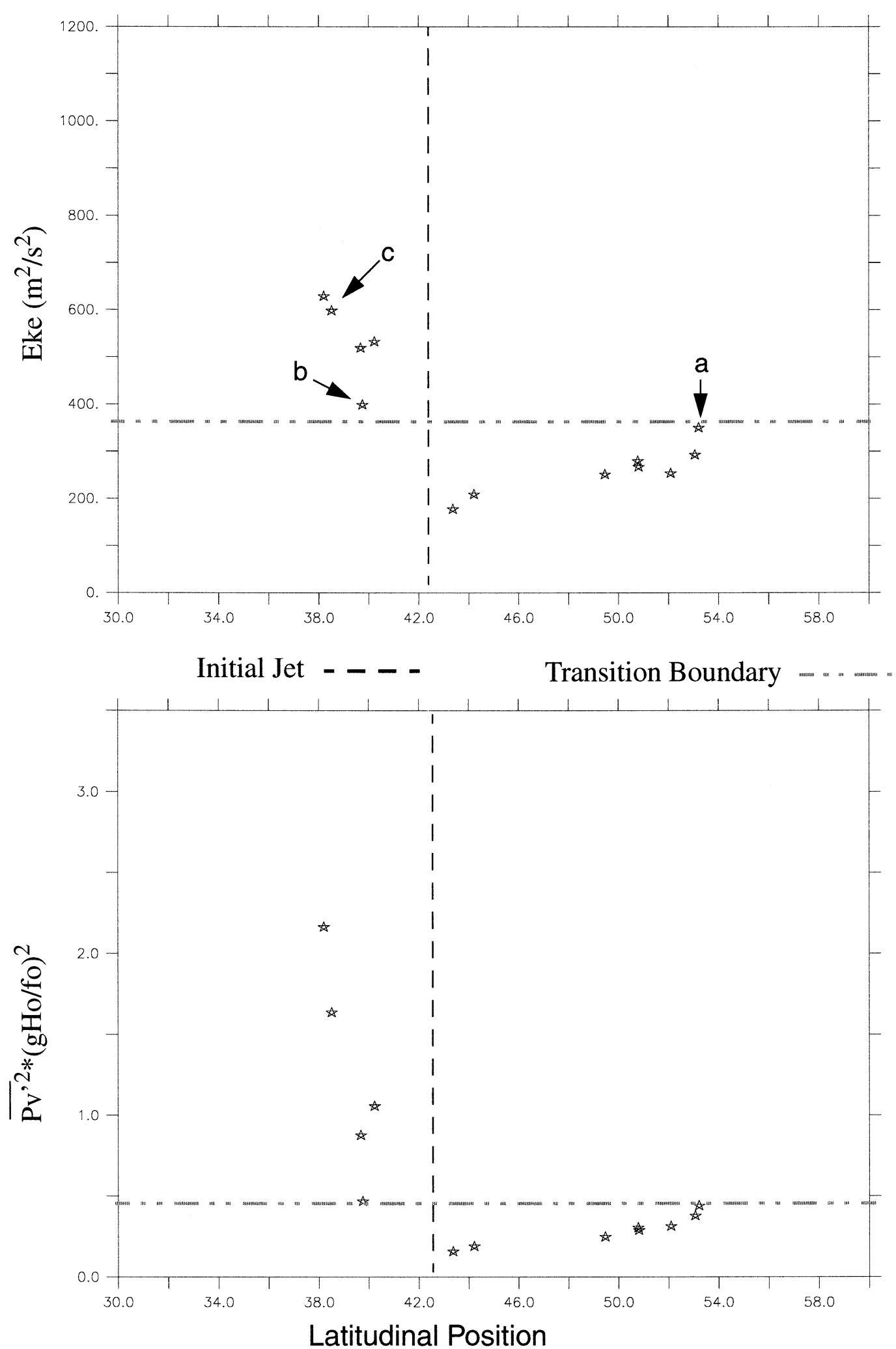

FIG. 13. Scatter diagram of the EKE and square of the PV anomaly maximum for all cases with $m=7$ vs the final position in latitude of the zonal jet maximum. The latitudinal position of the initial jet maximum is indicated by a dashed line and the level where the abrupt transition occurs is shown with a dotted-dashed line. 


\section{Characteristics of the permanent cyclone solutions CVC $(m=7, c=24 \mathrm{~m} / \mathrm{s}, \mathrm{W}=769 \mathrm{~m}$, Eke597)}

Potential Vorticity vs. Stream Function

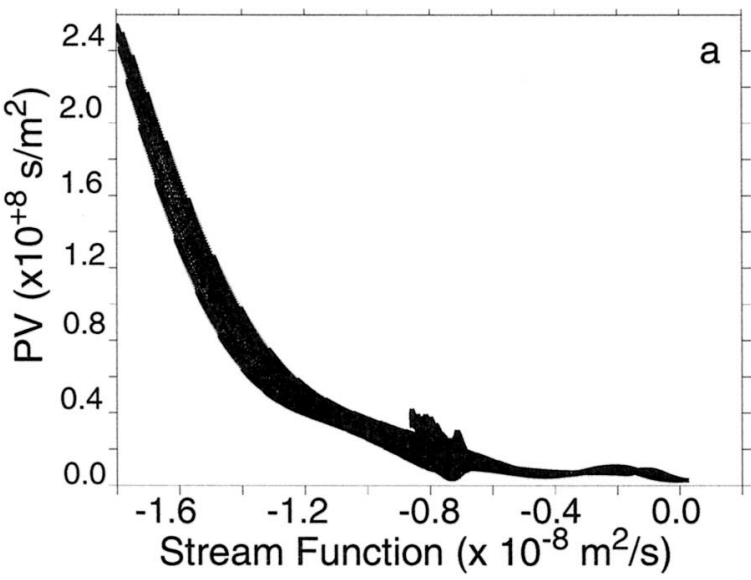

Zonal Potential Vorticity ( $\mathbf{(})$ and PV anomaly (*)
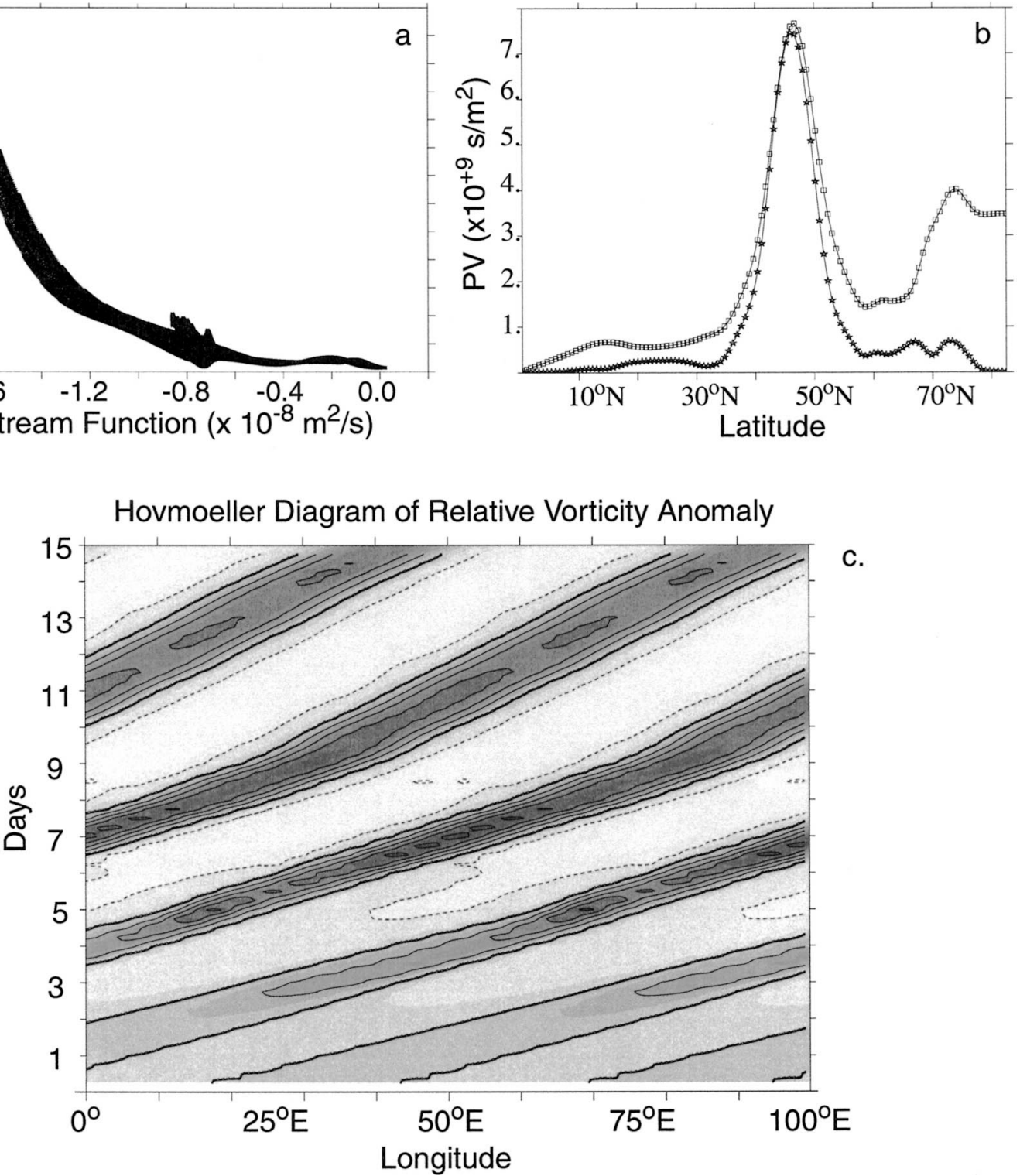

FIG. 14. (a) Scatter diagram of PV vs streamfunction for the solution marked $c$ in Fig. 13 (see text). (b) The profiles of the final zonally averaged PV and SPV [see Eq. (4.5)] for the corresponding solution. (c) The Hovmoeller diagram of the relative vorticity.

the solution. We will address this point later. Figure 14b shows the corresponding PV profile for solution c, averaged over the same last 7 days and the square root of the averaged potential enstrophy defined as

$$
\mathrm{SPV}=\left[\overline{\left(\mathrm{PV}^{\prime}\right)^{2}}\right]^{0.5},
$$

where the bar and brackets denote zonal and time av- erage. The very intense solution $\mathrm{c}$ with permanent eddies displays a very striking characteristic in which both the zonal and eddy PV symmetrizes, equilibrating at the same level. This is a characteristic of all the permanent eddy solutions found. The PV for those cases can be expressed as

$$
\mathrm{PV}=Q(y)\left[1+q\left(x-C_{\mathrm{ph}} t\right)\right],
$$


where $Q(y)$ is the same functional form for the zonal $\mathrm{PV}$ and the eddies, $q$ is a function with zero zonal average, and

$$
\left(\overline{q^{2}}\right)^{0.5}=1 .
$$

Let us now discuss the role of $C_{\mathrm{ph}}$ from Eq. (4.4). Since $C_{\mathrm{ph}}$ does not have an apparent relation with the forcing phase velocity $c$, we derived the velocity $C_{\mathrm{ph}}$ by means of inspecting the Hovmoeller diagram of the relative vorticity anomaly of the solution denoted by point c in Fig. 13a. The Hovmoeller diagram is shown in the lower graph of Fig. 14. An estimated value of $C_{\mathrm{ph}} \sim$ $9.8 \mathrm{~m} \mathrm{~s}^{-1}$ can be obtained from the Hovmoeller diagram in the latter days of the life cycle. We have done the scatterplot for PV versus $\Psi+C_{\mathrm{ph}} y$ for different values of the phase velocity $C_{\mathrm{ph}}$ and the best fit was for a value of $C_{\mathrm{ph}}$ around $10 \mathrm{~m} \mathrm{~s}^{-1}$, which was very close to the previously estimated value from the Hovmoeller diagram. It should be noted that the forcing propagation speed for this case was $c=24 \mathrm{~m} \mathrm{~s}^{-1}$. The conclusion is that if the eddies are strong enough to redistribute the total PV, it will modify the environment to such an extent that it will find a particular steering level on the redistributed zonal flow. Although the topic is fascinating, it will distract us from our primary topic and research on these issues will continue.

\section{Waves $m=5,6,7$, and 8}

\section{a. The eddy kinetic energy for all the modes}

In order to understand that strong interactions, discussed in the previous section, are not only peculiar to a particular horizontal scale $(m=7)$, let us discuss the solutions for all the wavenumbers studied $(m=5,6$, 7 , and 8). The final latitudinal position of the jet maximum versus the maximum EKE for the four modes studied, including the recently discussed $m=7$ is shown in Fig. 15 (similar to the upper graph of Fig. 13). Most of the solutions display similar characteristics in relation to the maximum eddy kinetic energy and the position of the jet. For low values of EKE, the jet is positioned poleward of the initial position; as the eddy energy increases, the jet is displaced further poleward. After surpassing a critical value of EKE, the jet position jumps to the equatorward side of the initial position. This abrupt transition is due to a bifurcation that occurred in the life cycle of the eddy, as previously discussed for $m=7$. Note that this critical value depends on the mode being considered.

Here, $\mathrm{EKE}_{\text {critical }}=200 \mathrm{~m}^{2} \mathrm{~s}^{-2}$ for $m=8 ; \mathrm{EKE}_{\text {critical }}=$ $375 \mathrm{~m}^{2} \mathrm{~s}^{-2}$ for $m=7$; EKE $\mathrm{E}_{\text {critical }}=700 \pm 30 \mathrm{~m}^{2} \mathrm{~s}^{-2}$ for $m=6$, and $\mathrm{EKE}_{\text {critical }}=1500 \pm 50 \mathrm{~m}^{2} \mathrm{~s}^{-2}$ for $m=5$. A similar behavior could be seen if PV (not shown) had been used instead of EKE. Below the threshold energy for each mode, AVC is predominant and around the critical value for EKE there is a transition zone. In this transition zone, the eddies' large amplitudes and small perturbations may shift the instability to one control or the other. But, as the energy of the eddies increases, it settles into a CVC system. These results strongly suggest that two extremes may exist; when EKE $<\mathrm{EKE}_{\text {critical }}$, $\mathrm{AVC}$ dominates, and if $\mathrm{EKE}>\mathrm{EKE}_{\text {critical }}$, $\mathrm{CVC}$ dominates.

\section{b. The transition zone}

In order to understand better the transition from AVC to CVC, let us inspect two solutions indicated by the arrows in Fig. 15 [for convenience CVC $(m=6)$ and AVC $(m=6)]$. Both have very similar energy levels $\left(\right.$ EKE $\left.\sim 694 \mathrm{~m}^{2} \mathrm{~s}^{-2}\right)$. The only difference in generating each solution was the propagation speed for the forcing $c=12 \mathrm{~m} \mathrm{~s}^{-1}$ for CVC $(m=6)$ and $c=24 \mathrm{~m} \mathrm{~s}^{-1}$ for $\operatorname{AVC}(m=6)$. The evolution of CVC $(m=6)$ and AVC $(m=6)$ for two times in their life cycle is shown in Fig. 16. The relative vorticity and wind vectors for days 4 and 7 are shown in Fig. 17. It should be stressed that the initial evolution is quite similar. By day 4 , both waves look very much alike. However, by day 7 both solutions are bifurcating, one to a CVC and the other to an AVC. It seems that the speed of the initial wave is a perturbation to the finite-amplitude vortex interaction to make the abrupt jump, but as previously stressed, it is not due to a resonance mechanism. Note also that all the solutions with CVC do not have similar final states. Some display an undulating PV whereas the others consist of a distinct streak of eddies. This seems to confirm the previous discussion of $m=7$, that only when the eddies are able to redistribute the PV and find a compatible steering level, can they survive as individual entities. Figure 17 corroborates the fact that CVC $(m=6)$ produces a jet equatorward of the initial position and AVC $(m=7)$ produces a jet poleward of the initial position. The eddy kinetic energy is also shown for both cases in Fig. 17. CVC $(m=6)$ produces eddies that remain longer but are not quite as permanent as in the $m=7$ case, whereas AVC zonalizes very quickly.

\section{c. The bifurcation diagram}

It is useful to think that the eddy energy level may be the indicator for classifying AVC and CVC, as well as when a poleward shift or equatorward shift can be expected for the zonal flow. Figure 18a tries to represent the eddy kinetic energy for all the cases showing whether they are CVC (+) or AVC (-) as a function of wavenumber. The dashed curve crudely represents values of $\mathrm{EKE}_{\text {critical }}$ that we estimated as a function of $(m)$. Although a careful inspection will show few (-) AVC points above the curve, most of the points consistently appear above the $\mathrm{EKE}_{\text {critical }}$ to be CVC (+). Consequently, the jet will shift equatorward whereas below the curve will be predominately AVC (-) and the jet will shift poleward. Representing the same result in a slightly different way, Fig. 18b attempts to summarize 

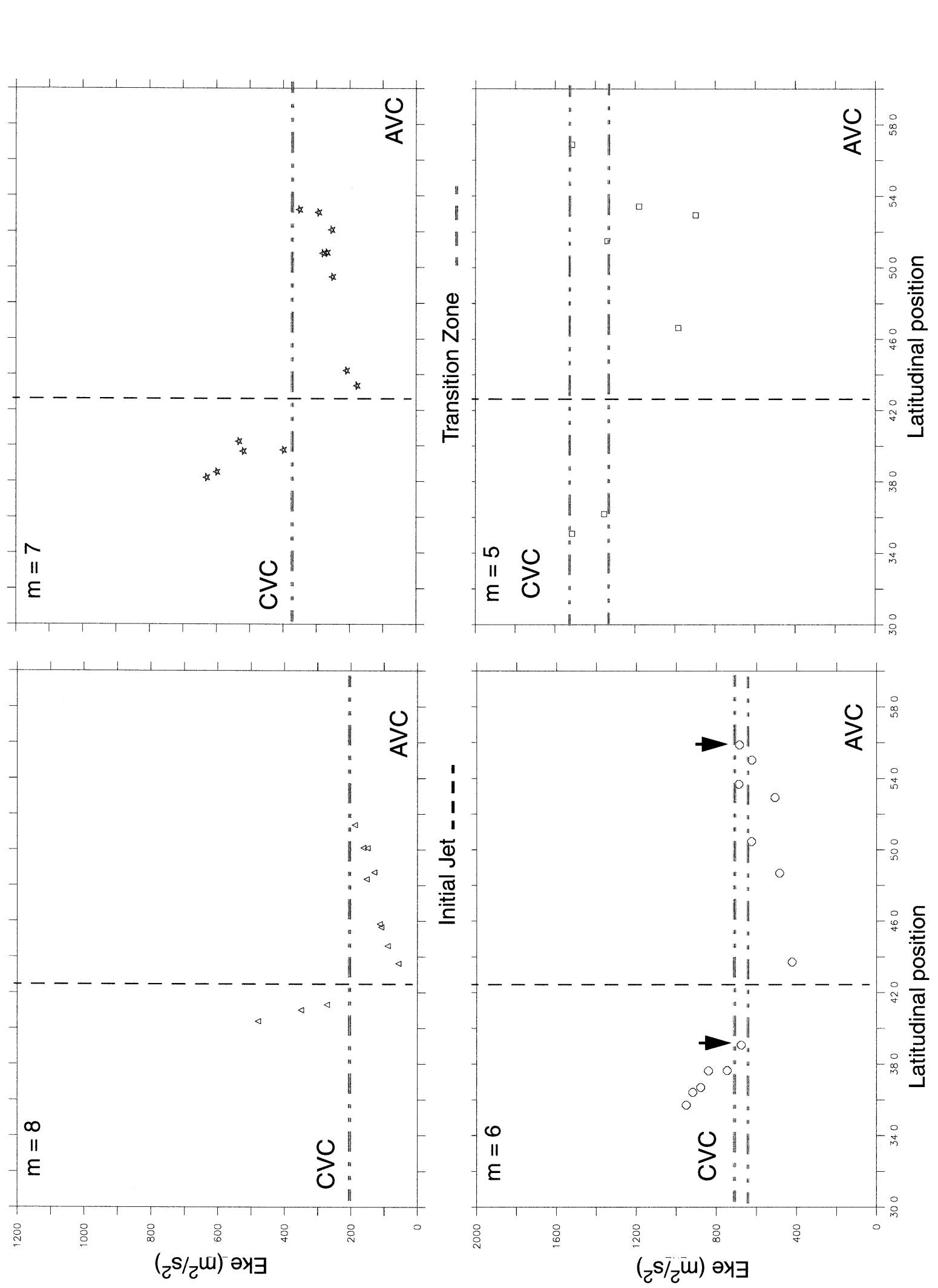


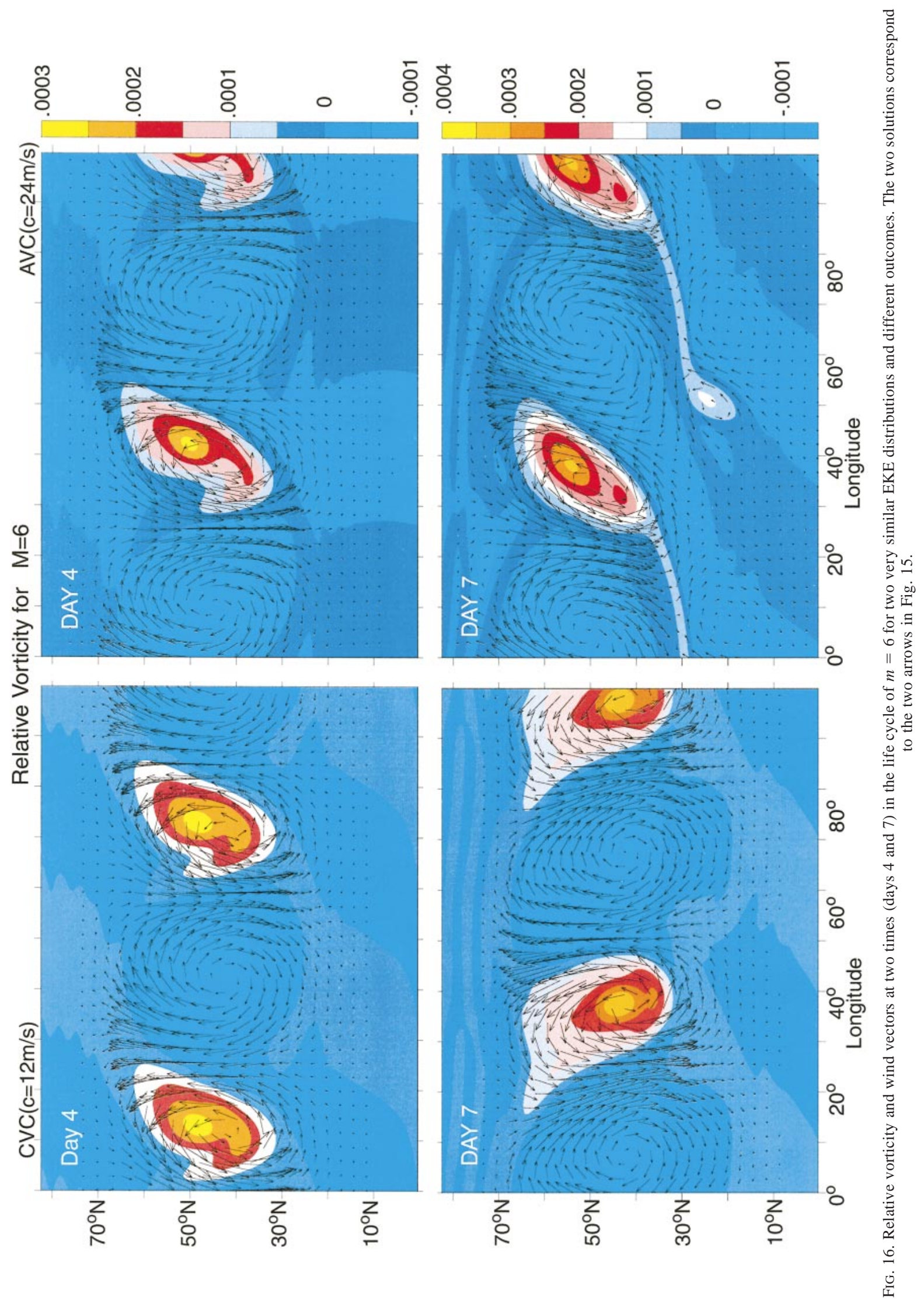



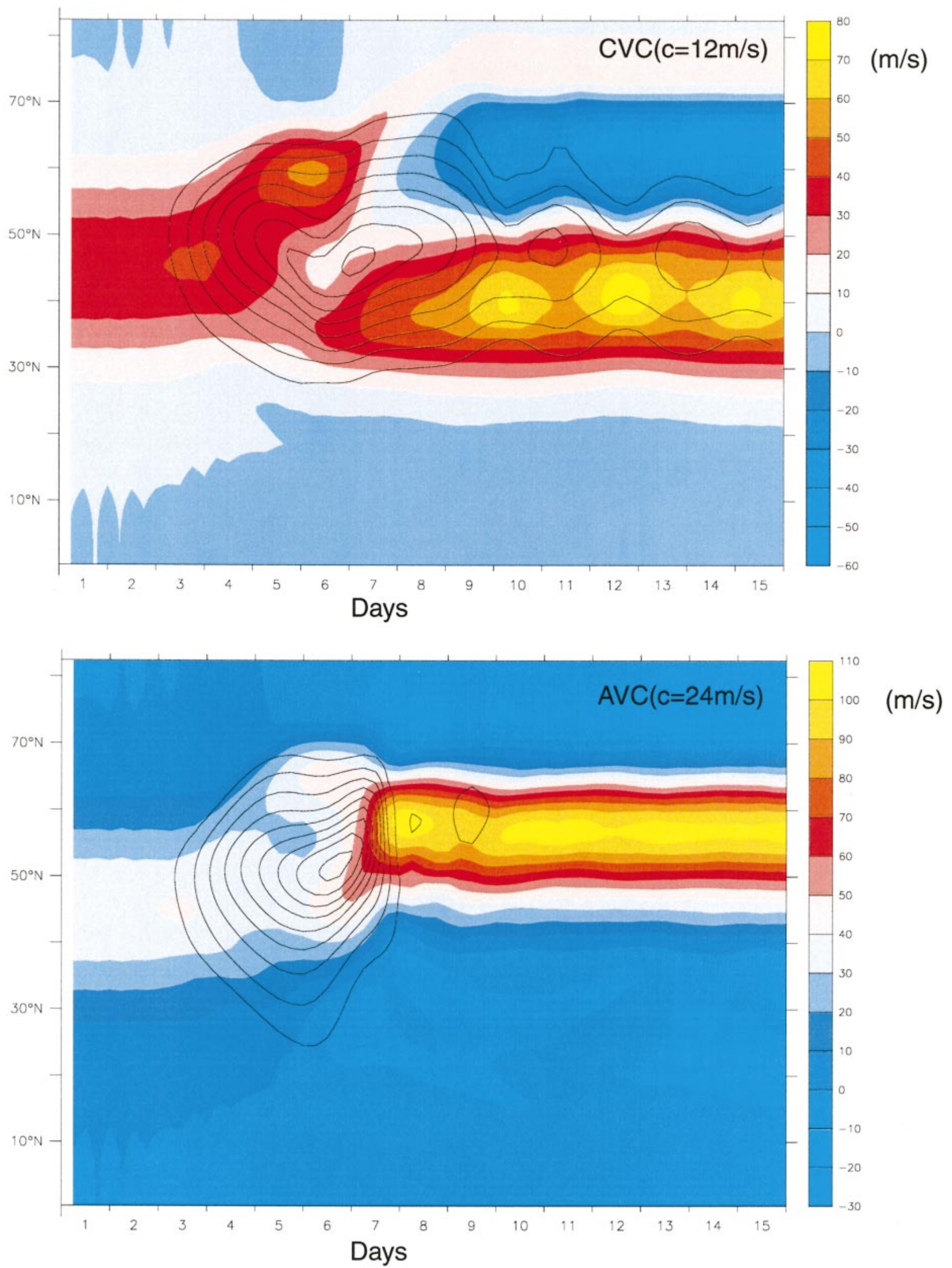

FIG. 17. Similar to Fig. 12. The time evolution of the zonal mean flow and the eddy kinetic energy for these two $m=6$ solutions. 

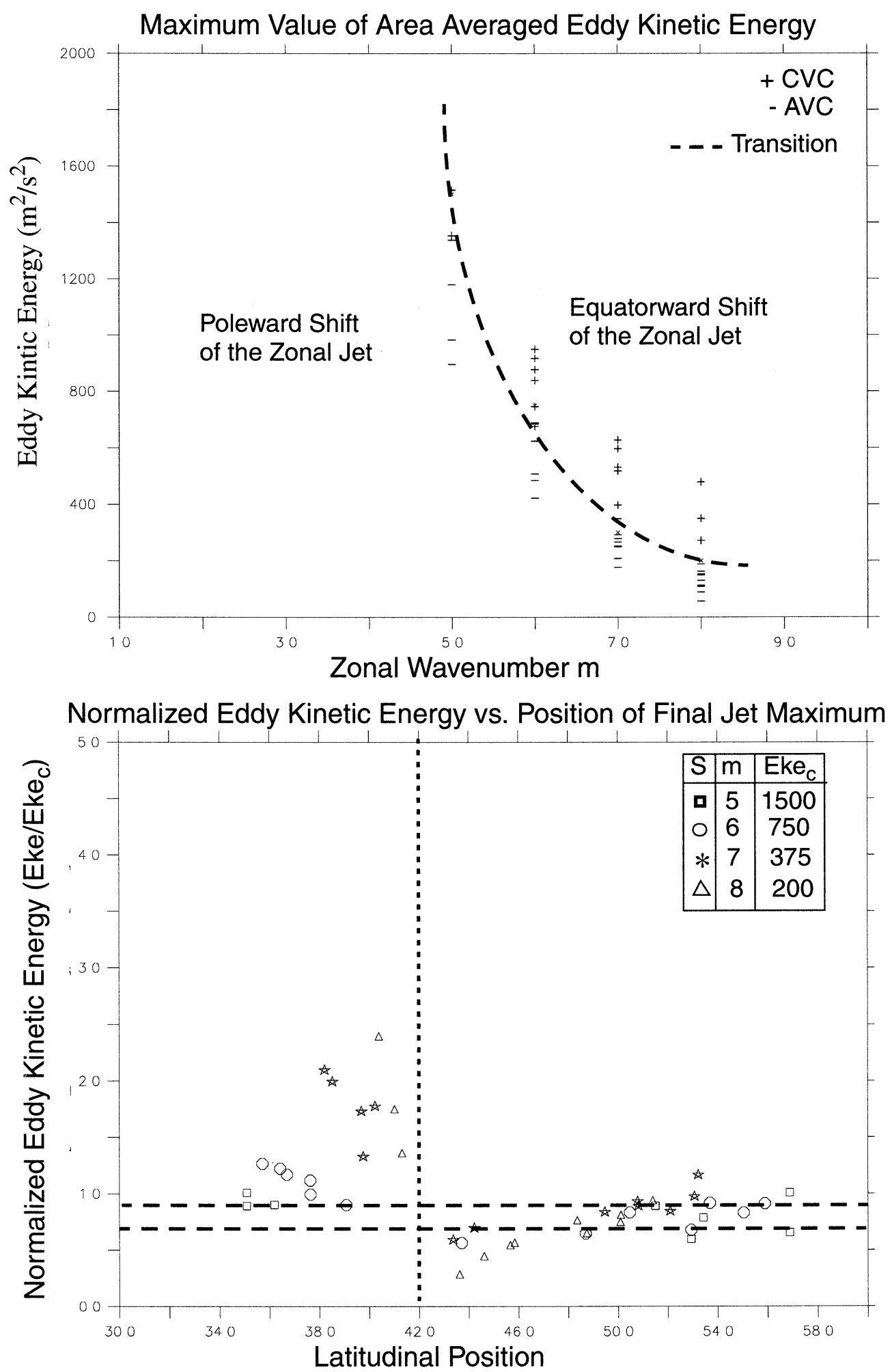

FIG. 18. (a) The stability diagram for the finite-amplitude wave breaking EKE as a function of wavenumber. The dashed curve is the $\mathrm{EKE}_{\text {critical }}$ values, $(+)$ indicates a CVC case, and the (-) indicates an AVC case. Lower eddy energy causes the jet to be displaced to the poleward side, whereas high energies cause the opposite to occur; the jet is displaced to the equatorward side. (b) The unified diagram of all cases for all the wavenumbers of eddy energy normalized by its corresponding $\mathrm{EKE}_{\text {critical }}$ as a function of latitudinal position of the final zonal jet maximum. The values of the symbols and $\mathrm{EKE}_{\text {critical }}$ used are in the table insert. The two dashed lines indicate, as before, the transition zone (around unity) and the dotted line indicates the position of the initial jet. 
all the results in a single graph. The eddy kinetic energy of all the wavenumbers normalized by each individual $\operatorname{EKE}_{\text {critical }}(m)$ is shown versus the latitudinal displacement of the final jet. The symbols show the different wavenumbers, and the two dashed lines indicate the transition zone around unity. The table indicates the $\operatorname{EKE}_{\text {critical }}(m)$ values used for normalizing the respective solutions.

\section{Summary and conclusions}

By analyzing a number of very high resolution, nonhydrostatic experiments of baroclinic life cycles, we came to the conclusion that the intensity of the nearsurface baroclinic development influences the upperlevel wave to such an extent that it could produce cyclonic or anticyclonic wave breaking. Since the final jet position is equatorward or poleward, the position depends on whether the waves break cyclonically or anticyclonically, respectively. The low-level baroclinicity plays a very important role in the outcome of the wave and feedback to the mean circulation. Using a low-order shallow water model, we test the hypothesis that the intensity of the eddy forcing from the lower layers of the atmosphere can have a profound effect on the disturbances of the upper layers.

To that end we use two different versions of a global

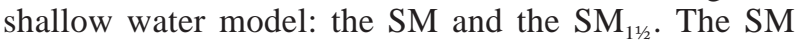
is the shallow water model in which the height is relaxed to a forcing that simulates the stretching effects of a lower-level unstable wave. The $\mathrm{SM}_{1 \frac{1}{2}}$ on the other hand, is a PV conserving model in which the forcing is prescribed to a lower-level interface. The bulk of the results were done with the SM, but a few solutions of the $\mathrm{SM}_{1 \frac{1}{2}}$ were used to verify the same behavior. From these experiments we conclude the following.

- The intensity of the lower-level baroclinicity (forcing amplitude) seems to be a determining factor for the quality of upper-level wave breaking.

- For weak intensities, the strong effective beta asymmetries due to the earth's sphericity produce anticyclonic wave breaking and a poleward shift of the zonal jet will occur.

- For moderate forcing, anticyclonic wave breaking occurs and consequently, as before, a poleward shift of the zonal jet will occur. However, there is an important distinction between weak and moderate forcing. In the latter case, the eddy anticyclonic centers are very intense. These vortex centers are intense enough (AVC) to produce a difluence in the cyclonic centers, squelching the cyclone in the NW-SE direction and elongating it in the other two corners; this process has two effects: thinning the cyclone and producing positive vorticity fluxes on the north, negative fluxes on the south, and moving the jet poleward.

- By increasing the forcing, the cyclone centers become considerably more intense than the anticyclones
(CVC) and they are able to deform and thin the anticyclones, thus moving the jet equatorward. This transition is very abrupt (Fig. A2) above a threshold amplitude, the life cycle bifurcates to a cyclonic wave breaking.

Let us now return to the point raised at the beginning in the introduction, that is, the possibility that baroclinic eddies can indeed produce such a response on the jet axis. This question is related to what kind of feedback this bifurcation of the eddy life cycle can be expected to produce on the storm track variability. We can then speculate about the relation of the quality of wave breaking to the variations in space and time of the storm tracks. The implications for storm track variability are quite direct. In normal years, at the entrance of the storm track intense baroclinicity produces CVCs with a slight shift of the jet equatorward. At the last half of the storm track, due to much weaker baroclinicity, anticyclonic wave breaking occurs (AVCs) displacing the jet poleward. The eddies at the entrance of the storm track develop from the baroclinicity of the subtropical jet. Downstream fluxing and weaker surface baroclinicity makes the upper-level waves more aloft and barotropic by the middle of the storm track. These waves normally break anticyclonically, enhancing the subpolar eddydriven jet. In the warm phase of ENSO, more baroclinicity (and subtropical moisture flux) is present in the eastern Pacific Ocean. This enhanced baroclinicity could support more CVCs in the eastern basin, maintaining the subtropical jet farther east. It is clear the that these processes are of weekly timescale. Eddies initiate around the subtropical latitudes; if the surface baroclinicity is weak, they deflect the jet poleward due to the anticyclonic breaking producing a subpolar eddy-driven jet (Orlanski 1998). In periods of enhanced subtropical baroclinicity, the eddies will continue breaking cyclonically along the whole storm track causing the subtropical jet to extend farther east. Certainly there are other possibilities. For instance, the subtropical baroclinicity is very weak all over, and only very modest amounts of eddy energy are generated along the storm track; the subtropical jet will also be extended to the eastern $\mathrm{Pa}$ cific Ocean. The caveat of the argument, when moderate to high eddy energies are generated, is of course, the assumption that waves always break. However, if waves could decay by other means, like wave-wave interaction or downstream propagation (Chang and Orlanski 1993), locally, it would weaken the feedback to the mean circulation. Although the baroclinic eddy life cycle should have been in the environment of the storm track rather than single-wave evolution, the general case is beyond the scope of this study. We are presently performing high-resolution storm track experiments to be able determine unambiguously the role of wave-mean flow interactions.

Acknowledgments. I would like to thank Isaac Held for providing me with the shallow water global model 
and discussions that helped clarify the research, and to Guillaume Lapeyre for familiarizing me in the use of the model. I would also like to thank Steve Garner for the development of the ZETANC model used in the three-dimensional simulations. The useful comments provided by Steve Garner and Geoffrey Vallis were gratefully appreciated. Finally, I would also like to thank Larry Polinsky for editing the manuscript.

\section{APPENDIX}

\section{The 11/2-Layer Shallow Water Model $\left(\mathrm{SM}_{1 \frac{1}{2}}\right)$}

We consider a model of the upper layers of the troposphere of density $\rho_{1}$ that overlies a lower layer with density $\rho_{2}$ and below that, a layer at rest with $\rho_{0}$ as shown in Fig. A2b. These layers should be viewed as the atmosphere contained between two isentropessay, 400 and $310 \mathrm{~K}$, shown in Fig. A2a. Figure A2a also shows the initial wind profile and potential temperature profiles of the initial value calculations shown in Figs. 2 and 3. Basically, we can assume the stratosphere to be the upper layer at rest, the upper troposphere is the dynamically active layer, and we have a passive lower layer where the forcing is applied that mimics the eddy activity of the lower layer. A simpler scheme would have been to consider a reduced gravity shallow water model with one layer and a topography that varies as a function of space and time. The pressure in the configuration that we utilized is given as follows:

$$
\begin{array}{ll}
P_{0}=g \rho_{0} H-g \rho_{0} z, & H>z>\left(H-h_{1}\right), \\
P_{1}=g \rho_{1}\left(h_{1}-z\right)+P_{0}=g \Delta \rho_{1} h_{1}+g \rho_{0} H-g \rho_{1} z, & \left(H-h_{1}\right)>z>(H \\
P_{2}=g \rho_{2}\left(h_{2}-z\right)+P_{1}=g \Delta \rho_{2} h_{2}+g \Delta \rho_{1} h_{1}+g \rho_{0} H-g \rho_{2} z, & \left(H-h_{2}\right)>z,
\end{array}
$$

where $\Delta \rho_{i}$ is the density jump from layers 1 and 2 . The basic steady state is in geostrophic balance and using the expression for the pressures from (A.1),

$$
\left(f+\frac{\bar{U}_{1} \tan \phi}{a}\right) \bar{U}_{1}=-\frac{1}{a \rho_{1}} \frac{\partial \bar{P}_{1}}{\partial \phi}=-\frac{g_{1}^{\prime}}{a} \frac{\partial \bar{h}_{1}}{\partial \phi} ;
$$

and the lower-layer height is

$$
\begin{aligned}
\left(f+\frac{\bar{U}_{2} \tan \phi}{a}\right) \bar{U}_{2} & =-\frac{1}{a \rho_{2}} \frac{\partial \bar{P}_{2}}{\partial \phi} \\
& =-\frac{g_{2}^{\prime}}{a} \frac{\partial \bar{h}_{2}}{\partial \phi}-\frac{\rho_{1}}{\rho_{2}} \frac{g_{1}^{\prime}}{a} \frac{\partial \bar{h}_{1}}{\partial \phi},
\end{aligned}
$$

where the reduced gravity in each layer is $g_{i}^{\prime}=g \Delta \rho_{i} /$ $\rho_{i}$. Substituting the last term of (A.3) with (A.2), the slope of the lower interface is given by the Margules relation:

$$
\begin{aligned}
(f & \left.+\frac{\bar{U}_{2} \tan \phi}{a}\right) \bar{U}_{2}-\frac{\rho_{1}}{\rho_{2}}\left(f+\frac{\bar{U}_{1} \tan \phi}{a}\right) \bar{U}_{1} \\
= & -\frac{g_{2}^{\prime}}{a} \frac{\partial \bar{h}_{2}}{\partial \phi} .
\end{aligned}
$$

Note that since the Boussinesq approximation has not been made, the density in the lower-most layer could be very large compared to the middle layer. In particular, if $\rho_{2}$ goes to infinity, $h_{2}$ is considered a rigid surface and since $g_{2}^{\prime} \sim 1$ and $U_{2}=0$, no geostrophic balance requirements are made for the slope of the bottom topography in this hydrostatic mode. If $\rho_{2}$ is much larger than $\rho_{1}$, but finite like the deep layer of Jupiter, $h_{2}$ could have a slope to balance wind in that deep layer (Dowling 1993). We crudely try to represent the vertical shear of the baroclinic profile; assuming that $U_{1}$ and $U_{2}$ have a similar jet structure, but $U_{2}$ has a smaller amplitude than $U_{1}$, (A.4) can be written approximately as follows:

$$
d\left(f+\frac{2 d \bar{U}_{1} \tan \phi}{a}\right) \bar{U}_{1}=\frac{g_{2}^{\prime}}{a} \frac{\partial \bar{h}_{2}}{\partial \phi},
$$

where $1-d=U 2 / U 1$ is a measure of the vertical shear, the quadratic term in $d$ was neglected in Eq. (A.5).

The $1 \frac{1}{2}$-layer shallow water equation for the sphere may be written as

$$
\begin{gathered}
\frac{\partial u_{1}}{\partial t}-\left(\zeta_{1}+f\right) v_{1}=-\frac{1}{a \cos \phi} \frac{\partial}{\partial \lambda}\left(g_{1}^{\prime} h_{1}+K_{1}\right), \\
\frac{\partial v_{1}}{\partial t}+\left(\zeta_{1}+f\right) u_{1}=-\frac{1}{a} \frac{\partial}{\partial \phi}\left(g_{1}^{\prime} h_{1}+K_{1}\right), \\
\frac{\partial}{\partial t}\left(h_{1}-h_{2}\right)+\nabla \cdot\left[\left(h_{1}-h_{2}\right) \mathbf{v}_{1}\right]=0,
\end{gathered}
$$

where $\lambda, \phi$, and $t$ are the longitude, latitude, and time, respectively; $a$ is the earth's radius; $u$ and $v$ are the zonal and meridional velocities; $K_{1}=|\mathrm{v}|^{2} / 2$ is the kinetic energy per unit mass; $\zeta=\operatorname{curl}(\mathbf{v}$ is the relative vorticity; and $f=2 \Omega \sin \phi$ is the Coriolis parameter.

Since our intent is to simulate upper-level waves that are generated by stretching from a growing low-level unstable baroclinic wave, it will be enough to prescribe a time-space-dependent interface $h_{2}(\lambda, \gamma, t)$ in (A.8) and calculate the evolution of the variables of layer 1 by integrating the system (A.6)-(A.8). Here, $h_{2}$ can be 


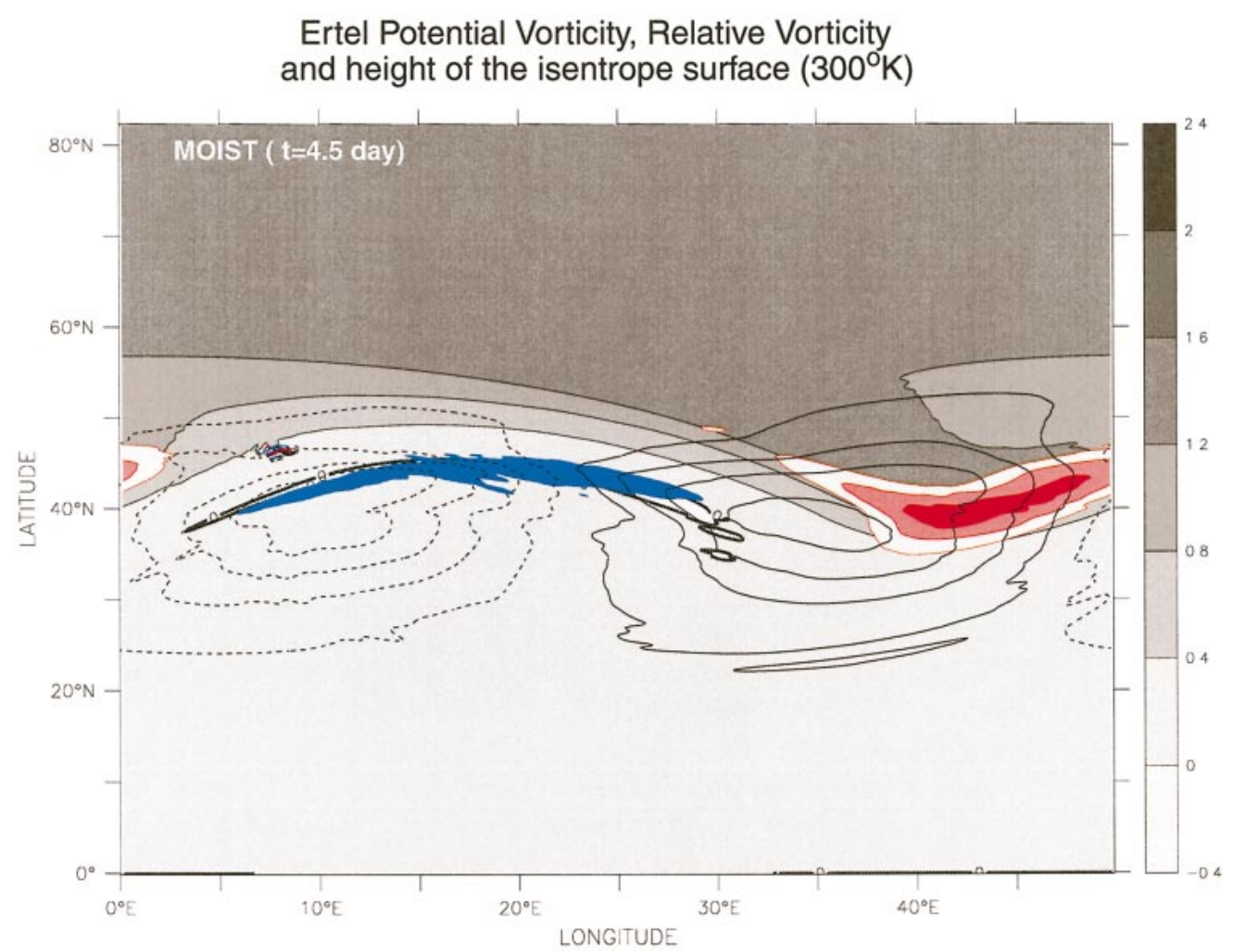

Fig. A1. The snap shot at day 4.5 for the moist experiment (shown in Figs. $2 \mathrm{~b}$ and $3 \mathrm{~b}$ ) for the PV at the $300-\mathrm{K}$ isentrope (PV unit $=$ $10^{-6} \mathrm{~K}\left(\mathrm{~m} \mathrm{~s}^{-1}\right.$ ), the anomaly height of the $300-\mathrm{K}$ isentrope (interval $=500 \mathrm{~m}$ ), and the vertically averaged relative vorticity anomaly between 5000 and $10000 \mathrm{~m}$ (interval $=0.25 \times 10^{-4} \mathrm{~s}^{-1}$ ).

viewed as a time-dependent bottom topography. However, in this derivation it seems more justified to use a mean slope that is in geostrophic balance with a prescribed zonal velocity in the lower layer. It seems clear that regardless of the prescribed forcing in $h_{2}$, the system (A.6)-(A.8) conserves the layer potential vorticity:

$$
\mathrm{PV}=\frac{\zeta+f}{h_{1}-h_{2}}
$$

Without losing generality let us assume that the reduced gravity in layer 1 and 2 are the same. Then multiplying (A.8) by $g^{\prime}$ and redefining $g^{\prime} h_{1}=h_{1}$ and $g^{\prime} h_{2}$ $=h_{2}$, we can eliminate the index 1 from all the variables:

$$
\mathrm{PV}=\frac{\zeta+f}{h-h_{2}} .
$$

The potential vorticity in (A.10) has the same form as in (A.9), the new $h$ represents the geopotential height, and from now on we will use the geopotential as the new prognostic variable in the model. All the influence from the lower layer, similar to that shown in Fig. A1 for the midlevel height of the isentrope, will be prescribed through $h_{2}$ :

$$
h_{2}=\bar{h}_{2}(\phi)+W_{0}\left(\frac{t}{t_{\mathrm{LF}}}\right)^{2} \exp \left[-\left(\frac{t}{t_{\mathrm{LF}}}\right)^{2}\right] \frac{U i(\phi)-c}{U_{0}-c} \cos \left[m_{0}(\lambda-c t]\right. \text {, }
$$

where the first term on the rhs is given by (A.4) for different values of $d$ and the second term in rhs portrays a growing wave with wavenumber $m_{0}$ phase speed $\mathrm{c}$ and a modulation in amplitude with timescale $t_{\mathrm{LF}}$. This forcing is the same as in (3.2)-(3.5), the relaxation function for $h$ in the Shallow Water Model described in section 3. Although the functions for the forcing are similar in both models, the Shallow Water Model (SM) and the 11/2-layer Shallow Water Model $\left(\mathrm{SM}_{1 \frac{1}{2}}\right)$, there are considerable differences between them. The $\mathrm{SM}_{1 \frac{1}{2}}$ conserves potential vorticity, whereas the SM, due to the Newtonian forcing, does not, at least for the period 


\section{Zonal Mean Flow and Potential Temperature for the Baroclinic Simulations}

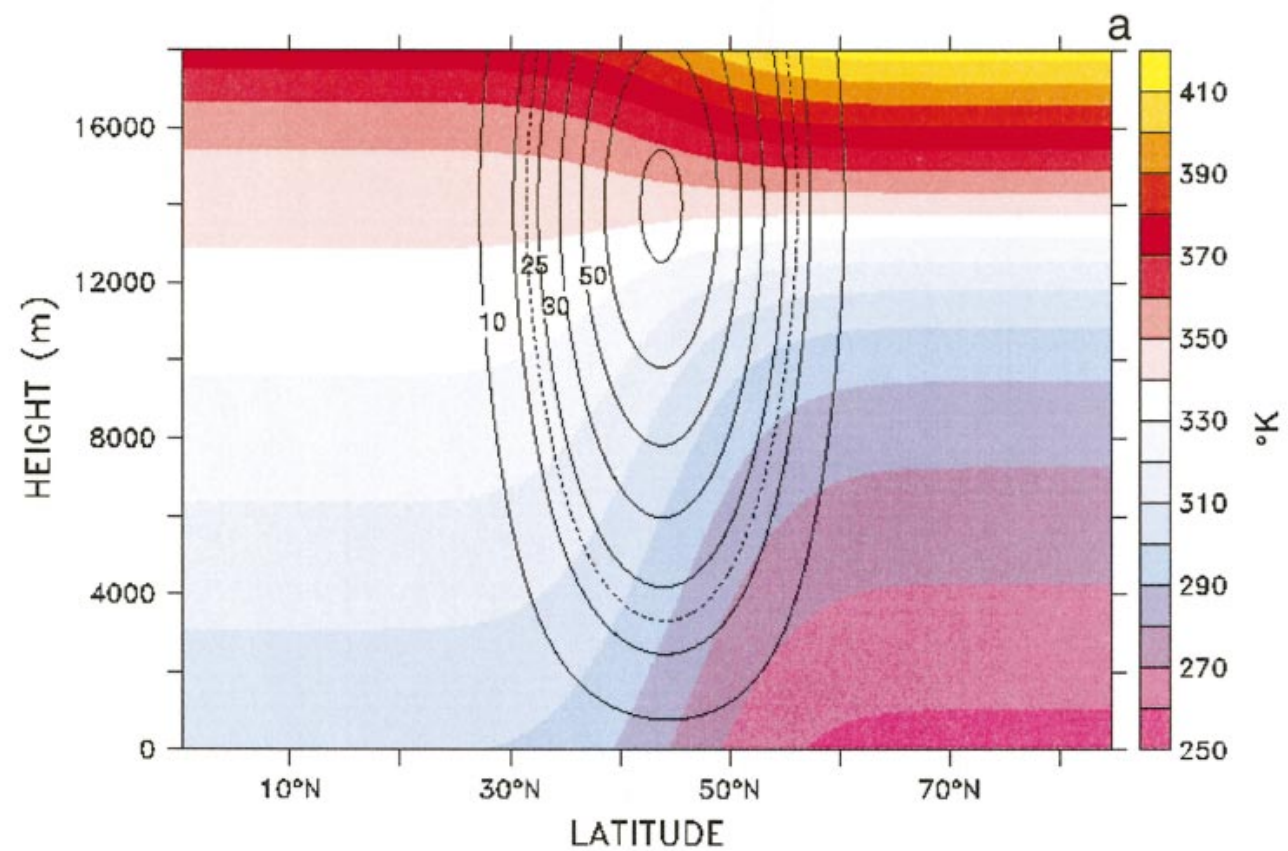

The Shallow Water Model $\mathrm{SM}_{1-1 / 2}$

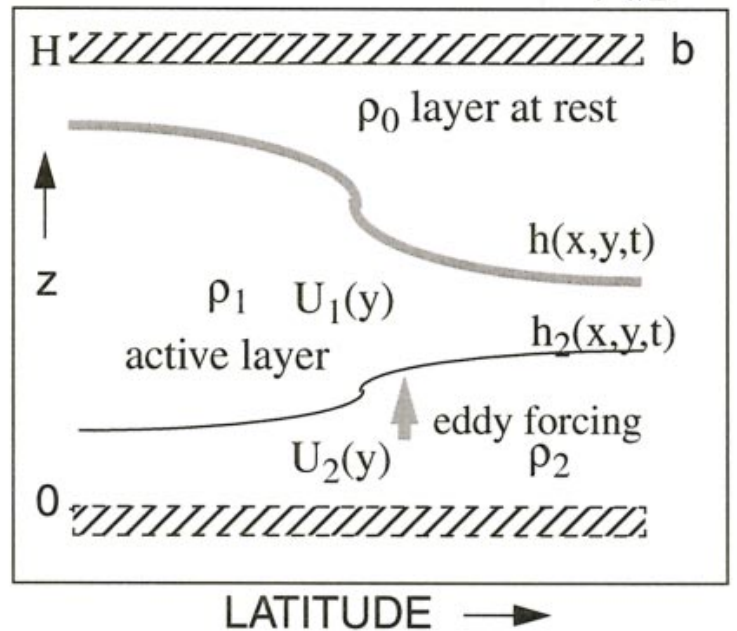

The Shallow Water Model SM

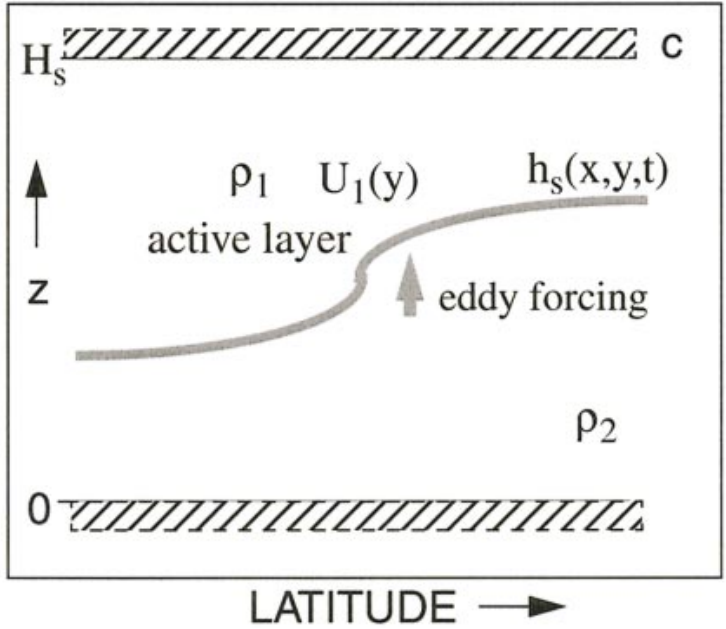

FIG. A2. (a) Characteristic of the initial potential temperature (color bands) and zonal jet (black contour) used in the threedimensional solutions (Figs. 2 and 3). (b) Characteristics of the $\mathrm{SM}_{1 \frac{1}{2}}$ and (c) the SM (see text).

in which the forcing is acting. Moreover, SM depends on a relaxation timescale, whereas $\mathrm{SM}_{1^{1 / 2}}$ does not. These seem to be two limitations of SM. However, a major limitation that $\mathrm{SM}_{1 \frac{1}{2}}$ has is the fact that it reacts freely to a forcing like bottom topography. It radiates Rossby waves from the region where the forcing is applied to both poleward and equatorward sides of it. This is a quite expected response; however it is not what unstable baroclinic waves will do. In order for baroclinic waves to become unstable there should be a phase lock between the upper and lower wave disturbances preventing the Rossby-wave radiation, at least in its growth phase. Actually, when a lower breaking wave occurred, usually before the upper-level wave, the upper-level wave being freed from its lower-level anchor could radiate as Rossby waves to both sides of the unstable region. Held (2000) discussed the meridional circulation resulting from the topographic forcing in a shallow water model, 

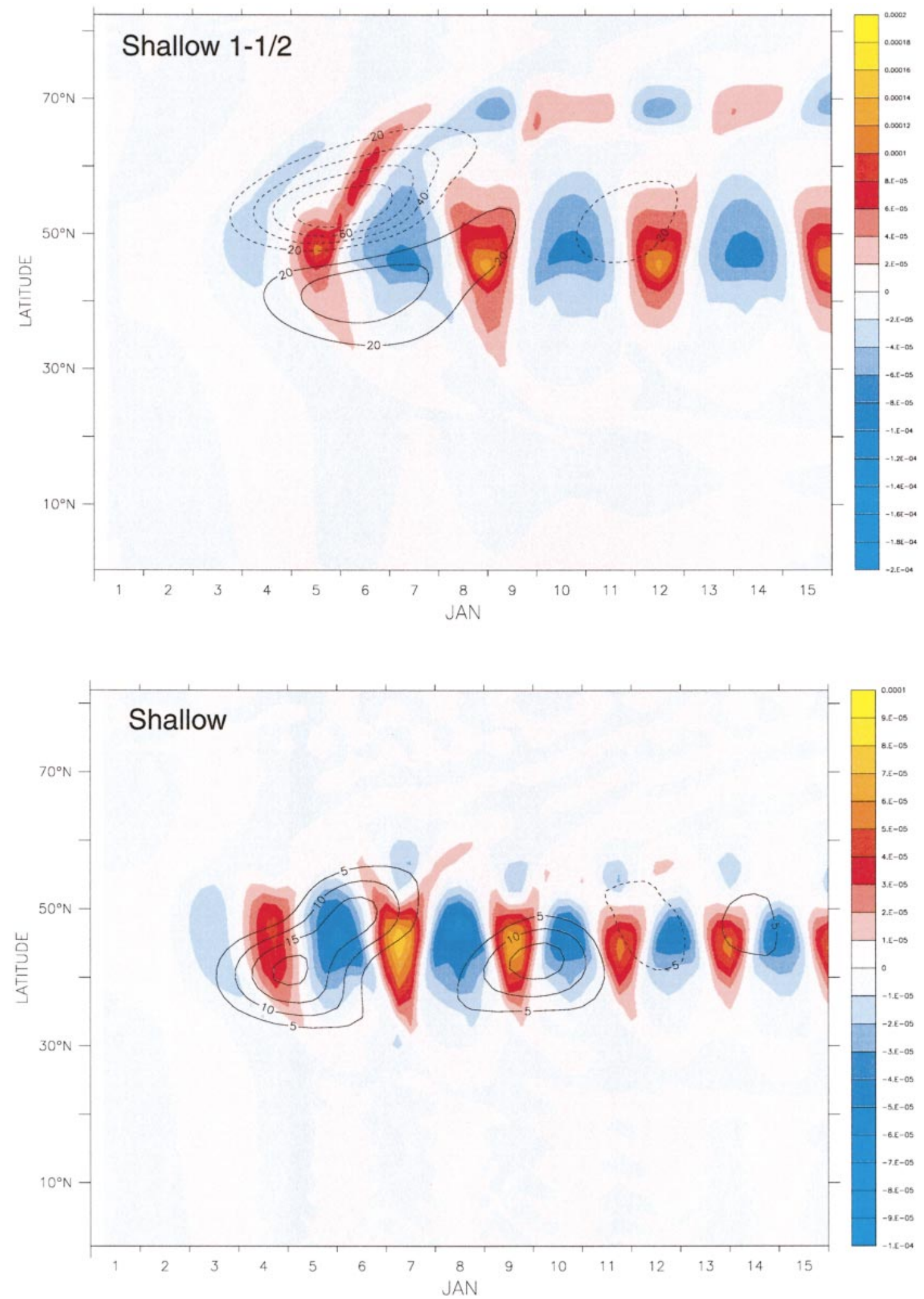

FIG. A3. Hovmoeller diagram of the relative vorticity at $x=45^{\circ}$ longitude (color bands) and meridional momentum flux (black contour) for the $\mathrm{SM}_{1 \frac{1}{2}}$ and $\mathrm{SM}$. Note that the intervals are different for both cases. 

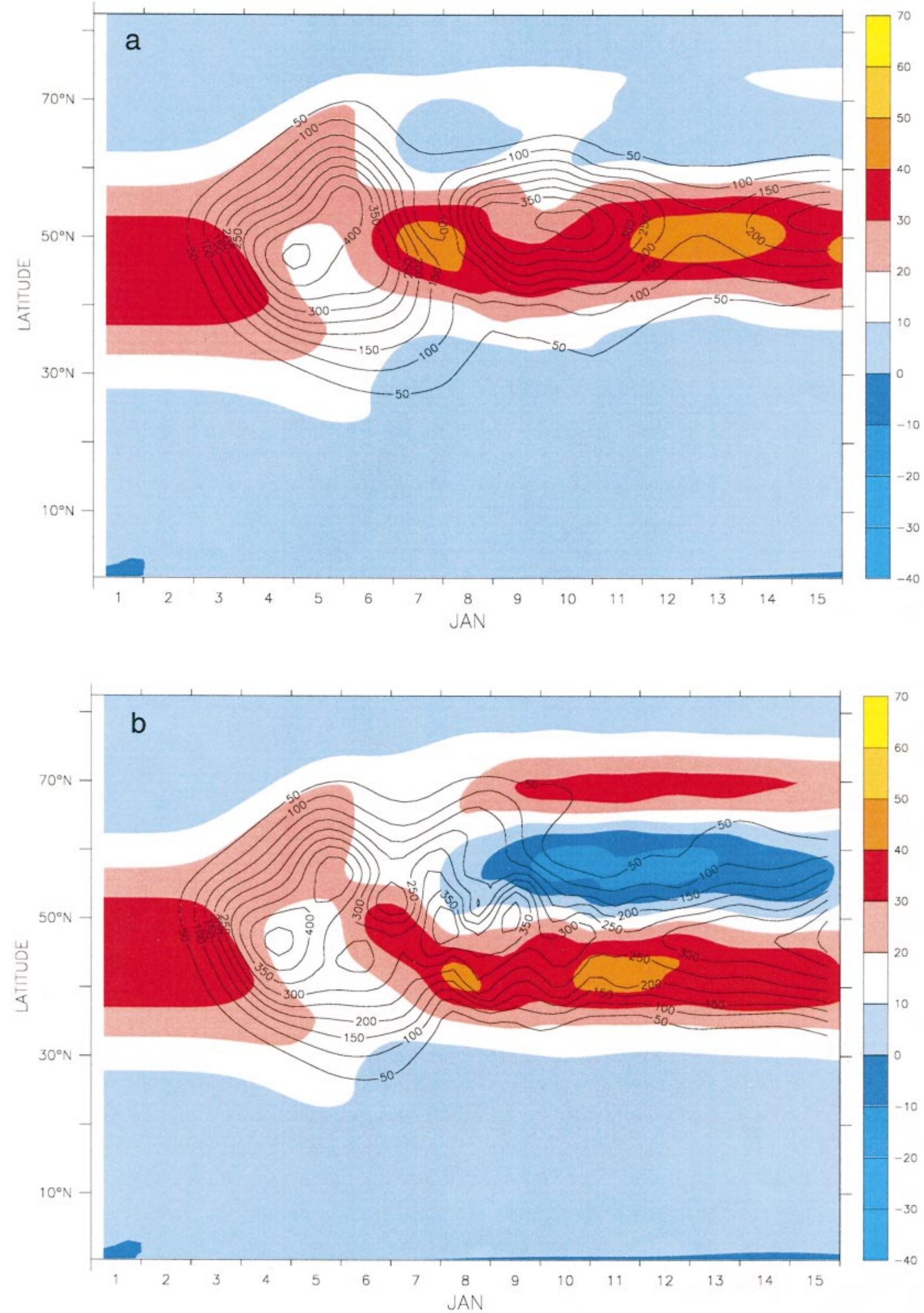

FIG. A4. The zonal mean flow evolution over the 15 days and the EKE corresponding to two solutions of the $\mathrm{SM}_{1^{1 / 2}}$ is shown. Compare this figure with the evolution using SM in Fig. 12. 
pointing out that in a statistical steady state it will be similar to the meridional circulation produced by baroclinic disturbances:

$$
\overline{v^{\prime} q^{\prime}}=\overline{v^{\prime} \zeta^{\prime}}-f_{0} \frac{\overline{v^{\prime} h^{\prime}}}{H_{0}} \approx 0 .
$$

Furthermore, if it is assumed that no wave breaking occurs in the topography region, the potential vorticity flux vanishes. To paraphrase Held (2000), "Rossby waves radiating from a source region transport zonal momentum into this region. Therefore, between the source region, the momentum flux convergence must be balanced by an eddy mass flux:

$$
f_{0} \frac{\overline{v^{\prime} h^{\prime}}}{H_{0}}=-\frac{\partial}{\partial y} \overline{u^{\prime} v^{\prime}} .
$$

It follows that the eddy mass flux should be poleward above the topography. Since there cannot be total mass flux, the mass transport by the 'mean meridional circulation', $v$, must be equatorward just as in the uppertropospheric branch of the Farrel Cell." Although this is true, the timing when the circulation is produced in the $\mathrm{SM}_{1^{1 / 2}}$ and in the baroclinic development is different. In $\mathrm{SM}_{1 \frac{1}{2}}$ the wave radiation is done soon when the forcing is applied, whereas, in the baroclinic development cases, is accomplished when the upper-level wave is breaking. To illustrate this effect, Fig. A3 shows the Hovmoeller diagram of the relative vorticity (at $x=45^{\circ}$ longitude) and meridional momentum fluxes (zonal averaged $u^{\prime} v^{\prime}$ ) for both the $\mathrm{SM}_{1_{1 / 2}}$ and SM. It is clear that $\mathrm{SM}_{1 \frac{1}{2}}$ displays radiation of Rossby waves as the disturbance is growing. This radiation of waves, as Held (2000) points out, will produce momentum fluxes into the jet. The response of the of SM is different in that respect, practically no radiation of Rossby waves is noticeable and the momentum flux is poleward. Note that the equations for $\mathrm{SM}$ and $\mathrm{SM}_{1 \frac{1}{2}}$ are the same if one makes the transformation $h=H_{s}-h_{s}$ and assumes $h_{2}$ $=0$.

\section{REFERENCES}

Balasubramanian, G., and S. T. Garner, 1997a: The role of momentum fluxes in shaping the life cycle of a baroclinic wave. J. Atmos. Sci., 54, 510-533.

— and $\longrightarrow, 1997 \mathrm{~b}$ : The equilibrium of short baroclinic waves. J. Atmos. Sci., 54, 2850-2871.

Berbery, E. H., and C. S. Vera, 1996: Characteristics of the Southern Hemisphere winter storm track with filtered and unfiltered data. J. Atmos. Sci., 53, 468-481.

Chang, E. K. M., 1993: Downstream development of baroclinic waves as inferred from regression analysis. J. Atmos. Sci., 50, 20382053.

— , and I. Orlanski, 1993: On the dynamics of a storm track. $J$. Atmos. Sci., 50, 999-1015.

Clough, S. A., N. S. Grahame, and A. O'Neill, 1985: Potential vorticity in the stratosphere derived using data from satellites. Quart. J. Roy. Meteor. Soc., 111, 335-358.
Dowling, T. E., 1993: A relationship between potential vorticity and zonal wind on Jupiter. J. Atmos. Sci., 50, 14-22.

Gross, B. D., 1997: The effect of compressibility on barotropic and baroclinic instability. J. Atmos. Sci., 54, 24-31.

Held, I. M., cited 2000: The general circulation of the atmosphere. Lecture Notes: Woods Hole GFD Summer School 2000. [Available online at http://gfd.whoi.edu/proceedings/2000/PDF/ lectures2000.pdf.]

—_ S. W. Lyons, and S. Nigam, 1989: Transients and the extratropical response to El Niño. J. Atmos. Sci., 46, 163-174.

Horel, J. D., and J. M. Wallace, 1981: Planetary scale atmospheric phenomena associated with the Southern Oscillation. Mon. Wea. Rev., 109, 813-829.

Hoskins, B. J., 1971: Atmospheric frontogenesis models: Some solutions. Quart. J. Roy. Meteor. Soc., 97, 139-153.

_- and D. J. Karoly, 1981: The steady linear response of a spherical atmosphere to thermal and orographic forcing. J. Atmos. Sci., 38, 1179-1196.

_- and P. J. Valdes, 1990: On the existence of storm-tracks. $J$. Atmos. Sci., 47, 1854-1864.

Lau, N.-C., 1985: Modeling the seasonal dependence of the atmospheric response to observed El Niños in 1962-1976. Mon. Wea. Rev., 113, 1970-1996.

Lee, S., and I. M. Held, 1993: Baroclinic wave packets in models and observations. J. Atmos. Sci., 50, 1413-1428.

Lim, G. H., and J. M. Wallace, 1991: Structure and evolution of baroclinic waves as inferred from regression analysis. J. Atmos. Sci., 48, 1718-1732.

McIntyre, M. E., and T. N. Palmer, 1984: The "surf zone" in the stratosphere. J. Atmos. Terr. Phys., 46, 825-849.

Nakamura, H., M. Nakamura, and J. L. Anderson, 1997: The role of high- and low-frequency dynamics in blocking formation. Mon. Wea. Rev., 125, 2074-2093.

Orlanski, I., 1998: On the poleward deflection of storm tracks. $J$. Atmos. Sci., 55, 128-154.

-, and J. J. Katzfey, 1991: The life cycle of a cyclone wave in the Southern Hemisphere. J. Atmos. Sci., 48, 1972-1998.

- and B. D. Gross, 2000: On the life-cycle of baroclinic eddies in a storm track environment. J. Atmos. Sci., 57, 3498-3513.

Polvani, L. M., D. W. Waugh, and R. A. Plumb, 1995: On the subtropical edge of the stratospheric surf zone. J. Atmos. Sci., 52, $1288-1309$.

Shapiro, M. A., H. Wernli, N. A. Bond, and R. Langland, 2001: The influence of the 1997-99 El Niño Southern Oscillation on extratropical baroclinic life cycles over the eastern North Pacific. Quart. J. Roy. Meteor. Soc., 127, 331-342.

Simmons, A. J., and B. J. Hoskins, 1978: The life cycles of some nonlinear baroclinic waves. J. Atmos. Sci., 35, 414-432.

—_, and _ 1979: The downstream and upstream development of unstable baroclinic waves. J. Atmos. Sci., 36, 1239-1254.

- , and -1980 : Barotropic influences on the growth and decay of nonlinear baroclinic waves. J. Atmos. Sci., 37, 1679-1684.

Sobel, A. H., and R. A. Plumb, 1999: Quantitative diagnostics of mixing in a shallow water model of the stratosphere. J. Atmos. Sci., 56, 2811-2829.

Thorncroft, C. D., B. J. Hoskins, and M. E. McIntyre, 1993: Two paradigms of baroclinic wave life-cycle behaviour. Quart. J. Roy. Meteor. Soc., 119, 17-55.

Trenberth, K. E., G. W. Branstator, D. Karoly, A. Kumar, N.-C. Lau, and C. Ropelewski, 1998: Progress during TOGA in understanding and modeling global teleconnections associated with tropical sea surface temperatures. J. Geophys. Res., 103, 14 291-14 324.

Webster, P. J., 1981: Mechanism determining the atmosphere response to sea surface anomalies. J. Atmos. Sci., 38, 554-571.

Whitaker, J. S., and C. Snyder, 1993: The effects of spherical geometry on the evolution of baroclinic waves. J. Atmos. Sci., 50, 597-612. 